\title{
Rubber friction: The contribution from the area of real contact
}

Cite as: J. Chem. Phys. 148, 224701 (2018); https://doi.org/10.1063/1.5037136

Submitted: 21 April 2018 . Accepted: 24 May 2018 . Published Online: 12 June 2018

A. Tiwari, N. Miyashita, N. Espallargas, and B. N. J. Persson

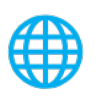

\section{ARTICLES YOU MAY BE INTERESTED IN}

Adhesion between rubber and glass in dry and lubricated condition

The Journal of Chemical Physics 148, 234702 (2018); https://doi.org/10.1063/1.5025605

The dependency of adhesion and friction on electrostatic attraction

The Journal of Chemical Physics 148, 144701 (2018); https://doi.org/10.1063/1.5024038

Theory of rubber friction and contact mechanics

The Journal of Chemical Physics 115, 3840 (2001); https://doi.org/10.1063/1.1388626

PHYSICS TODAY WHITEPAPERS
ADVANCED LIGHT CURE ADHESIVES

Take a closer look at what these

environmentally friendly adhesive systems can do
READ NOW

PRESENTED BY 8. MASTERBOND* 


\title{
Rubber friction: The contribution from the area of real contact
}

\author{
A. Tiwari, ${ }^{1,2}$ N. Miyashita, ${ }^{3}$ N. Espallargas, ${ }^{2}$ and B. N. J. Persson ${ }^{1, a)}$ \\ ${ }^{1}$ PGI-1, FZ Jülich, Jülich, Germany \\ ${ }^{2}$ Department of Mechanical and Industrial Engineering, Norwegian University of Science and Technology, \\ Richard Birkelandsvei 2B, N-7491 Trondheim, Norway \\ ${ }^{3}$ The Yokohama Rubber Company, 2-1 Oiwake, Hiratsuka, Kanagawa 254-8601, Japan
}

(Received 21 April 2018; accepted 24 May 2018; published online 12 June 2018)

\begin{abstract}
There are two contributions to the friction force when a rubber block is sliding on a hard and rough substrate surface, namely, a contribution $F_{\mathrm{ad}}=\tau_{\mathrm{f}} A$ from the area of real contact $A$ and a viscoelastic contribution $F_{\text {visc }}$ from the pulsating forces exerted by the substrate asperities on the rubber block. Here we present experimental results obtained at different sliding speeds and temperatures, and we show that the temperature dependency of the shear stress $\tau_{\mathrm{f}}$, for temperatures above the rubber glass transition temperature $T_{\mathrm{g}}$, is weaker than that of the bulk viscoelastic modulus. The physical origin of $\tau_{\mathrm{f}}$ for $T>T_{\mathrm{g}}$ is discussed, and we propose that its temperature dependency is determined by the rubber molecule segment mobility at the sliding interface, which is higher than in the bulk because of increased free-volume effect due to the short-wavelength surface roughness. This is consistent with the often observed reduction in the glass transition temperature in nanometer-thick surface layers of glassy polymers. For temperatures $T<T_{\mathrm{g}}$, the shear stress $\tau_{\mathrm{f}}$ is nearly velocity independent and of similar magnitude as observed for glassy polymers such as PMMA or polyethylene. In this case, the rubber undergoes plastic deformations in the asperity contact regions and the contact area is determined by the rubber penetration hardness. For this case, we propose that the frictional shear stress is due to slip at the interface between the rubber and a transfer film adsorbed on the concrete surface. Published by AIP Publishing. https://doi.org/10.1063/1.5037136
\end{abstract}

\section{INTRODUCTION}

Rubber friction enters in many important applications such as tires, dynamical seals, wiper blades, and syringes. It has been studied for many years starting with pioneering work by Greenwood and Tabor, ${ }^{1}$ Schallamach, ${ }^{2}$ Roberts and Tabor, ${ }^{3}$ and Grosch. ${ }^{4,5}$ It was found that on dry surfaces, there are two contributions to the rubber friction coefficient, one from shearing the area of real contact (the adhesive contribution $\mu_{\mathrm{ad}}$ ) and one from the pulsating deformations of the rubber surface due to the substrate asperities (the viscoelastic contribution $\mu_{\mathrm{visc}}$ ). The latter contribution depends on the internal friction of the rubber.

Detailed theories for the viscoelastic contribution to rubber friction have been developed by Klüppel and Heinrich ${ }^{6,7}$ and by Persson. ${ }^{8,9}$ The theory of Persson has been found to be in good agreement with exact numerical studies. ${ }^{10,11}$ Theories have also been developed for the adhesive contribution to rubber friction, but this topic is more complex and it appears that several processes may contribute, such as rubber stick-slip processes at the molecular level, opening crack propagation at asperities, rubber wear, plastic deformations, and plowing, the relative importance of which depends on the sliding velocity and the temperature.

Recently, Tiwari et al. ${ }^{12}$ presented experimental results for rubber friction on sandblasted glass substrates. For this

a)URL: www.MultiscaleConsulting.com. system, it was concluded that the most important contribution came from molecular stick-slip events at the sliding interface, while opening crack propagation in asperity contact regions was shown to be less important, in contrast to the belief of some groups. ${ }^{13,14}$ Thus, no definitive consensus exists about the origin of the adhesive contribution to the rubber friction.

In his pioneering studies, Grosch was able to construct smooth master curves for the friction coefficient by shifting the segments $\mu(v)$, obtained at different temperatures, using the bulk viscoelastic shift factor. These results indicated that rubber friction has the same time and temperature dependency as the bulk rubber viscoelastic modulus. However, we have found that the adhesive contribution to the friction has a different temperature dependency than the viscoelastic modulus. ${ }^{15}$ For this reason, we believe that one should instead construct shear stress master curves $\tau_{\mathrm{f}}(v)$ for the contribution to the friction force from the area of contact.

In this paper, we present experimental results for filled styrene-butadiene rubber (SBR) and unfilled and filled hydrogenated nitrile butadiene rubber (HNBR) slid on a concrete surface. The measurements were performed at different sliding speeds and temperatures. We show that the temperature dependency of the shear stress $\tau_{\mathrm{f}}$, for temperatures above the rubber glass transition temperature $T_{\mathrm{g}}$, is weaker than that of the bulk viscoelastic modulus. The physical origin of $\tau_{\mathrm{f}}$ for $T>T_{\mathrm{g}}$ is discussed below, and we propose that its temperature dependency is determined by the rubber molecule segment mobility at the sliding interface, which is higher 
than in the bulk due to increased free-volume effect due to the short-wavelength surface roughness. This is consistent with the often observed reduction in the glass transition temperature in nanometer-thick surface layers of glassy polymers. ${ }^{16,17}$

For temperatures $T<T_{\mathrm{g}}$, the shear stress $\tau_{\mathrm{f}}$ is nearly velocity independent and of similar magnitude as observed for glassy polymers such as PMMA or polyethylene. In this case, the rubber undergoes plastic deformations in the asperity contact regions and the contact area is determined by the rubber penetration hardness. In the same limit, we propose that the frictional shear stress is due to slip at the interface between the rubber and a hydrocarbon film adsorbed on the concrete surface. The hydrocarbon film (of nanometer thickness or more) is assumed to result from the transfer of molecules from the rubber to the substrate surface (transfer film).

This paper is organized as follows: In Sec. II, we briefly review the different contributions to rubber friction focusing on what we believe are the most important contributions from the area of contact. In Sec. III, we describe the different experiments we have performed, and in Secs. IV and V we present the experimental friction results for the SBR and HNBR compounds, respectively. In Sec. VI, we present a discussion about rubber friction below the glass transition temperature and show results for the surface topography of rubber surfaces after sliding on the concrete surface at $T=-40^{\circ} \mathrm{C}$. Section VII contains a discussion and Sec. VIII the summary and conclusion. In Appendix A, we review the basic equations of the viscoelastic contact mechanics theory used in analyzing the experimental data. In Appendix B, we present the measured viscoelastic modulus of all the rubber compounds and the power spectrum of the concrete surface.

\section{RUBBER FRICTION}

There are several contributions to rubber friction, the relative importance of which changes with the sliding velocity and the temperature. Here we consider the sliding of a rubber block on a hard substrate with a randomly rough surface. We will assume that the friction force can be written as the sum of the viscoelastic contribution $F_{\text {visc }}$ and a contribution from the area of real contact, which we assume is proportional to the contact area $A$,

$$
F_{\mathrm{f}}=F_{\text {visc }}+\tau_{\mathrm{f}} A,
$$

where $\tau_{\mathrm{f}}$ is the frictional shear stress acting on the area of contact. The viscoelastic contribution to $F_{\mathrm{f}}$ is usually calculated assuming that $\tau_{\mathrm{f}}=0$, i.e., without including the viscoelastic contribution resulting from the (time-dependent) shear deformations of the rubber, due to the adhesive interaction between the rubber molecules and the substrate.

In this study, and in earlier studies, information about $\tau_{\mathrm{f}}$ was obtained by analyzing experiments. Thus, $\tau_{\mathrm{f}}$ was obtained from the measured friction data by first subtracting the calculated $F_{\text {visc }}$ from the measured friction force. But since $F_{\text {visc }}$ is calculated assuming $\tau_{\mathrm{f}}=0$, it is clear that one contribution to $\tau_{\mathrm{f}}$ will be due to the viscoelastic deformations of the rubber (on different length scales, i.e., not just on the length scale of the asperity contact regions observed at the highest magnification). It follows that the effective $\tau_{\mathrm{f}}$ in (1) may be larger than the frictional shear stress which would prevail in the contact regions when sliding on a perfectly smooth substrate surface, where the (rubber) asperity contact regions would be statically deformed and hence not give a viscoelastic contribution to the friction force. Since we do not have a perfectly smooth concrete surface, we cannot measure $\tau_{\mathrm{f}}$ directly in the present case. However, measurements on other smooth surfaces often give frictional shear stresses of similar magnitude as we deduce for the concrete and asphalt road surface. As an example, in Fig. 1, we show $\tau_{\mathrm{f}}$ for carbon black filled natural rubber sphere [with the root-mean-square (rms) roughness $\approx 1 \mu \mathrm{m}$ ] sliding on a smooth surface (rms roughness $\approx 6.5 \mathrm{~nm}$ ) of calcium fluorite. ${ }^{18}$ The shear stress is shown as a function of the temperature increase, which varied because of variations in the load, sliding speed, and sliding time. Note that $\tau_{\mathrm{f}} \approx 2-5$ $\mathrm{MPa}$ which is smaller by a factor of $\sim 2$ than what we typically observe on rough surfaces, such as concrete or asphalt road surfaces.

If we write the normal force as $F_{\mathrm{N}}=p_{0} A_{0}$, where $p_{0}$ is the nominal contact pressure and $A_{0}$ is the nominal contact area, we get

$$
\mu=\mu_{\mathrm{visc}}+\frac{\tau_{\mathrm{f}}}{p_{0}} \frac{A}{A_{0}}=\mu_{\mathrm{visc}}+\mu_{\mathrm{cont}} .
$$

The viscoelastic contribution arises from the pulsating forces acting on the rubber block from the substrate asperities. There are several processes which can contribute to the friction from the area of real contact:

(a) Adhesive processes involving rubber molecules, or patches of rubber, at the sliding interface performing bonding-stretching-debonding cycles.

(b) Opening crack propagation at the exit of asperity contact regions.

(c) Hard filler particles (typically carbon or silica particles) in the rubber scratching the counter surface.

(d) Shearing of thin (nanometer) contamination films, e.g., the transfer film from the rubber.

(e) Energy dissipation in wear processes, e.g., the energy to remove particles from the rubber surface, which involves breaking covalent bonds in the rubber.

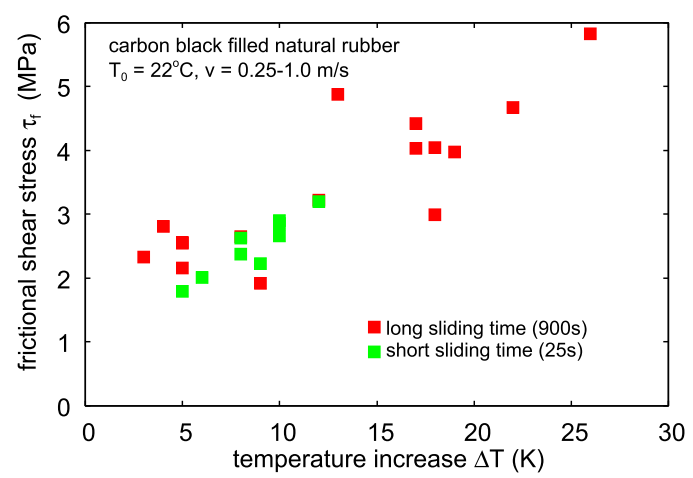

FIG. 1. The frictional shear stress $\tau_{\mathrm{f}}$ for carbon black filled natural rubber sliding on a smooth surface of calcium fluorite, as a function of the temperature increase $\Delta T$ due to frictional heating. The different data points correspond to sliding at different speeds (between $0.25 \mathrm{~m} / \mathrm{s}$ and $1 \mathrm{~m} / \mathrm{s}$ ), different loads (from $0.112 \mathrm{~N}$ to $1.012 \mathrm{~N}$, corresponding to the nominal contact pressures $0.57 \mathrm{MPa}$ to $2.22 \mathrm{MPa}$ ), and sliding time periods [ $\approx 900 \mathrm{~s}$ (red squares) and $\approx 25 \mathrm{~s}$ (green squares)]. Based on data from Ref. 18. 
(f) Plastic deformation and plowing, which will occur for temperatures below the glass transition temperature.

For sliding on clean dry surfaces, we believe that the viscoelastic contribution and the adhesive contribution (a) give the dominant contribution for most relevant sliding speeds and temperatures. However, at temperatures well below the rubber glass transition temperature, the viscoelastic contribution is not important and the most important mechanism appears to be associated with plastic deformation and shearing of thin transfer film from the rubber.

To illustrate the general nature of rubber friction on hard rough substrates, the circles in Fig. 2 show the master curve for the friction coefficient for unfilled HNBR sliding on a concrete surface. The master curve was obtained by shifting along the velocity axis the measured friction velocity segments $\mu(v)$ (with $v$ between $20 \mu \mathrm{m} / \mathrm{s}$ and $1 \mathrm{~mm} / \mathrm{s}$ ), obtained at many different temperatures (indicated in the figure), to form a smooth curve. In the figure, we also show the theoretically obtained viscoelastic contribution $\mu_{\mathrm{visc}}$ to the friction coefficient (green curve) and what we believe are the contributions from the area of contact from the adhesion process (a) denoted $\mu_{\mathrm{ad}}$ (pink curve) and from shearing a thin transfer film denoted $\mu_{\text {trans }}$ (red curve). We now briefly discuss the nature of the viscoelastic contribution and the contribution from the area of contact.

\section{A. Viscoelastic contribution to rubber friction}

When a rubber block is sliding on a hard rough substrate surface, the asperities of the substrate will exert timedependent deformations of the rubber. Since rubber materials have an internal friction or damping, the time dependent deformations will result in converting sliding kinetic energy into heat motion.

Surfaces of solids have roughness on very many different length scales, sometimes extending from macroscopic distances (say cm) down to atomic distances (say $\mathrm{nm}$ ), ${ }^{19}$ which all must be added together to obtain the total viscoelastic contribution to the friction force. This is illustrated in Fig. 3 where surface roughness is assumed to occur on only two length scales. Neglecting frictional heating, for surfaces which are self-affine fractal with the fractal dimension close to 2 (as

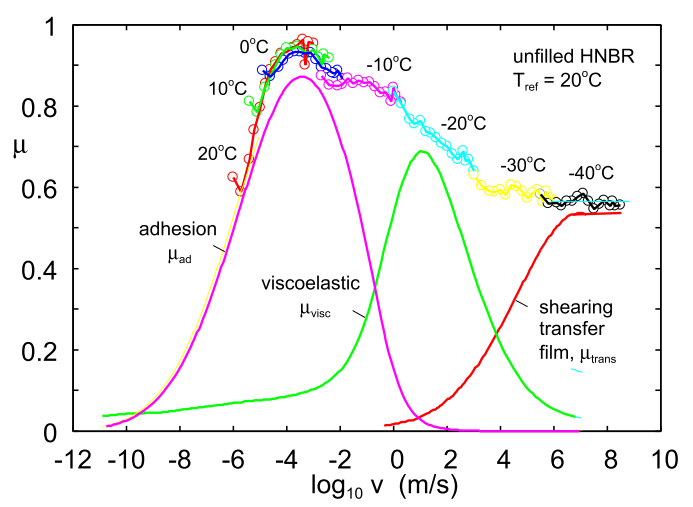

FIG. 2. Circles: master curve for $t=20^{\circ} \mathrm{C}$ for compound HNBR-0, obtained by shifting the velocity segments $\mu(v)$, obtained at different temperatures (between $-40{ }^{\circ} \mathrm{C}$ and $20{ }^{\circ} \mathrm{C}$ ), along the velocity axis to form as smooth curves as possible. Solid lines: the adhesion, viscoelastic, and transfer film contribution to the friction coefficient (see the text for details).

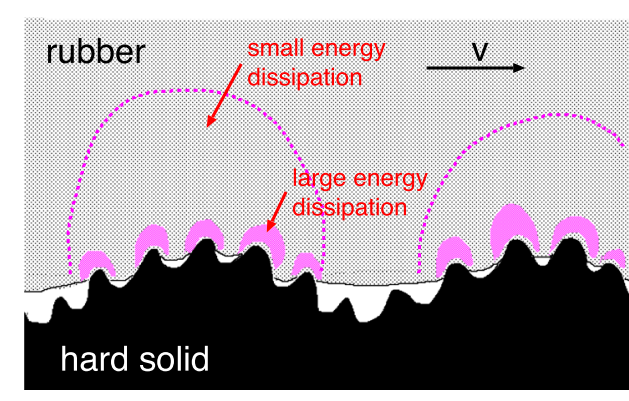

FIG. 3. The dissipated energy per unit volume is the highest in the smallest asperity contact regions. To get the total viscoelastic contribution to the friction, one must add up the contributions from roughness on all length scales.

is typical for road surfaces and other surfaces of practical importance, e.g., sandblasted surfaces), it has been shown that roughness on all length scales is roughly equally important, i.e., each decade in length scales contributes roughly equally to the total energy dissipated by the viscoelasticity of the rubber. ${ }^{20}$

The rubber viscoelastic modulus depends on the frequency $\omega$ and temperature $T$. At low frequencies (or high temperatures), rubber materials are soft with a typical Young's modulus of order $\sim 1 \mathrm{MPa}$ (rubbery region). At high frequencies (or low temperatures), it becomes very stiff with a Young's modulus of order $\sim 1 \mathrm{GPa}$ (glassy region). The transition region between the rubbery region and the glassy region is very important for rubber friction as in this frequency region the ratio between the imaginary part of the viscoelastic modulus (which is associated with energy dissipation) and the real part is large.

Since the viscoelastic contribution to rubber friction is determined by the bulk viscoelastic modulus, it will have the same temperature dependency as the viscoelastic modulus, which usually is described by the temperature-frequency shift factor $a_{T}$.

In analyzing the measured rubber friction data below, we have used the Persson theory for the viscoelastic contribution. ${ }^{9,10}$ In calculating $\mu_{\text {visc }}$ and the contact area $A$, it is necessary to use a large wavenumber (or short distance) cutoff $q_{1}$. We have found in the past ${ }^{9,21}$ that good agreement with experiments is obtained by choosing $q_{1}$ so that including all the roughness with wavenumbers smaller than $q_{1}$ results in a surface with the root-mean-square $(\mathrm{rms})$ slope $\approx 1$. For road surfaces, this usually corresponds to a cut-off length of order micrometer.

In analyzing the data presented below, we have chosen $q_{1}$ so that the rms slope equals 1.3 for the filled SBR and HNBR compounds and 0.85 for the unfilled HNBR compound; this gives good agreement with the experimental data. This difference in $q_{1}$ between filled and unfilled compounds may reflect the increased threshold tear strength of filled compounds as compared to unfilled compounds. ${ }^{22}$ Thus, we interpret $1 / q_{1}$ as the length scale where the stress at the interface becomes so large as to break covalent bonds in the rubber. The increased threshold tear strength of filled compounds will shift this length scale to shorter distances. For a discussion of the physical origin and interpretation of the cut-off $q_{1}$, see also Refs. 15 and 23 . 


\section{B. Adhesive contribution from the area of contact}

For the dry sliding contact between a rubber block and a substrate surface, in the asperity contact regions the rubber molecules are believed to undergo cyclic stick-slip events, as indicated in Fig. 4. In many cases, one expects weak interfacial interactions, e.g., van der Waals interaction. For stationary contact $(v=0)$, the rubber chains at the interface will adjust to the substrate potential to minimize the free energy. This bond formation may require overcoming potential barriers and will not occur instantaneously but requires some relaxation time. During sliding at low velocity, thermal fluctuations will help to break the rubber-substrate bonds resulting in a friction force which approaches zero as the sliding velocity goes to zero. At high velocity, there is not enough time for the rubber molecules to adjust to the substrate interaction potential, i.e., the bottom surface of the rubber block will "float" above the substrate forming an incommensurate-like state with respect to the corrugated substrate potential. Thus, the frictional shear stress is small also for large sliding speed. Hence we expect the frictional shear stress as a function of the sliding speed to have a maximum at some intermediate velocity $v_{0}^{*}$. This friction mechanism was first studied in a highly simplified model by Schallamach ${ }^{24}$ and later by Leonov et al. ${ }^{25}$ and for a more realistic model by Persson and Volokitin. ${ }^{26}$ The theory predicts that the frictional shear stress is a Gaussian-like curve as a function of $\log _{10} v$, with a width of 4 (or more) frequency decades and centered at a sliding speed typically of order $v_{0}^{*} \sim 1 \mathrm{~cm} / \mathrm{s}$; see the $\mu_{\text {ad }}(v)$ curve in Fig. 2. This frictional shear stress law is very similar to what was observed (measured) by $\mathrm{Grosch}^{4,5}$ for rubber sliding on smooth surfaces (glass or steel).

In Ref. 23, we found that using the following frictional shear stress law $\tau_{\mathrm{f}}(v, T)$ resulted in good agreement between theory and measurements:

$$
\tau_{\mathrm{f}}=\tau_{\mathrm{f} 0} \exp \left(-c\left[\log _{10}\left(\frac{v}{v_{0}^{*}}\right)\right]^{2}\right),
$$

where $c \approx 0.1, v_{0}^{*} \approx 6 \times 10^{-3} \mathrm{~m} / \mathrm{s}$, and $\tau_{\mathrm{f} 0} \approx 6.5 \mathrm{MPa}$. The full width at half maximum of the $\tau_{\mathrm{f}}(v)$ as a function of $\log _{10} v$ is $2(\ln 2 / c)^{1 / 2} \approx 5.3$.

The master curve (3) is for the reference temperature $T_{\text {ref }}=20^{\circ} \mathrm{C}$ and was obtained for a rubber with the glass transition temperature $T_{\mathrm{g} 0}=273-38 \mathrm{~K}$ (using the definition of $T_{\mathrm{g}}$ described below), but we have found that the frictional shear stress at other temperatures and for other compounds can be obtained approximately by replacing $v$ with $v a_{T}^{\prime}$, where
$a_{T}^{\prime}$ is the shift factor obtained when constructing the master curve (3),

$$
\ln a_{\mathrm{T}}^{\prime}=\frac{\epsilon}{k_{\mathrm{B}}}\left(\frac{1}{T}-\frac{1}{T_{0}}-\frac{1}{T_{\mathrm{g}}}+\frac{1}{T_{\mathrm{g} 0}}\right),
$$

where $T_{\mathrm{g}}$ is the glass transition temperature and $T_{0}=(273+20) \mathrm{K}$. The activation energy $\epsilon \approx 0.94 \mathrm{eV}$.

We have found that the parameters introduced above $\left(c, v_{0}^{*}, \tau_{\mathrm{f} 0}\right)$ for the adhesive contribution to the friction vary slightly depending on the studied system. Thus, for example, for $\tau_{\mathrm{f}}$, we used slightly different values for different compounds and road surfaces but usually in the range $\tau_{\mathrm{f} 0}=8 \pm 3 \mathrm{MPa}$. These values for $\tau_{\mathrm{f} 0}$ are similar but typically slightly larger than observed for very smooth surfaces (see Fig. 1 for one example). This may be partly due to a viscoelastic contribution to $\tau_{\mathrm{f} 0}$ on rough surfaces (see above) and also to the fact that on a rough substrate surface the rubber chain segments may be able to bind stronger at step edges and other defect sites, as compared to a perfectly flat substrate, as recently observed for polydimethylsiloxane rubber on smooth and slightly (atomically) roughened glass surfaces. ${ }^{27,28}$

We can write (3) and (4) in the following alternative form:

$$
\tau_{\mathrm{f}}=\tau_{\mathrm{f} 0} \exp \left(-c\left[\log _{10}\left(\frac{v}{v_{m}}\right)\right]^{2}\right)
$$

and

$$
v_{\mathrm{m}}=v_{0} \exp \left[-\frac{\epsilon}{k_{\mathrm{B}}}\left(\frac{1}{T}-\frac{1}{T_{\mathrm{g}}}\right)\right],
$$

where $v_{0}=v_{0}^{*} \exp \left[\left(\epsilon / k_{\mathrm{B}}\right)\left(1 / T_{0}-1 / T_{\mathrm{g} 0}\right)\right] \approx 5 \times 10^{-7} \mathrm{~m} / \mathrm{s}$. If we write $v_{0}=\lambda / \tau$ and assume a characteristic length of order a few nanometer, we get at the glass transition temperature the relaxation time $\tau \approx 0.01 \mathrm{~s}$. We believe that $\tau$ is the segmental relaxation time ( $\alpha$-relaxation process) for polymer chains in the surface region of the rubber block (see below).

The temperature dependency of the frictional shear stress is weaker than that of the bulk viscoelastic modulus. Thus the activation energy $\epsilon \approx 1 \mathrm{eV}$ we have found for the Arrhenius-like temperature dependency of $\tau_{\mathrm{f}}$ is only about half of the effective activation energy associated with the bulk viscoelastic modulus (as determined by the shift factor $a_{T}$ ). Similarly, the relaxation time $\tau$ is about 1000 times shorter than expected for the segmental relaxation time for the bulk rubber. We believe both effects are related to the fact that polymer chains at the rubber surface experience smaller energy barriers for segmental motion as compared to the bulk. ${ }^{16,17}$ Thus it is well known

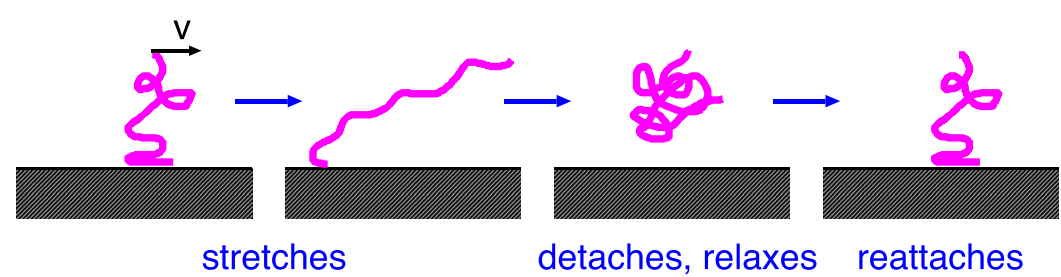

FIG. 4. The classical description of a polymer chain at the rubber-block counter surface interface. During lateral motion of the rubber block, the chain stretches, detaches, relaxes, and reattaches to the surface to repeat the cycle. The picture is schematic and in reality no detachment in the vertical direction is expected, but only a rearrangement of molecule segments (in nanometer-sized domains) parallel to the surface from pinned (commensurate-like) to depinned (incommensurate-like) domains. 
that the glass transition temperatures of polymer films decrease as the thickness decreases. In our case, we do not have a free surface but the rubber is in contact with a substrate surface, but because of surface roughness at short length scales and due to the sliding motion (see Fig. 5), it is likely that even in the area of contact there is a thin (nanometer) porous layer which reduced the energy barriers for segmental motion at the interface.

From the discussion above, one would expect that instead of (6) one should use

$$
v_{\mathrm{m}}=v_{0}^{\prime} \exp \left[-\frac{\epsilon}{k_{\mathrm{B}}}\left(\frac{1}{T}-\frac{1}{T_{\mathrm{g}}^{\prime}}\right)\right],
$$

where $T_{g}^{\prime}$ is an effective glass transition temperature at the interface. But we may still write $v_{\mathrm{m}}$ by the form (6) if we define

$$
v_{0}^{\prime}=v_{0} \exp \left[-\frac{\epsilon}{k_{\mathrm{B}}}\left(\frac{1}{T_{\mathrm{g}}^{\prime}}-\frac{1}{T_{\mathrm{g}}}\right)\right] .
$$

Experiments on polymer films with thickness $\sim 10 \mathrm{~nm}$ give typically glass transition temperatures $\approx 50 \mathrm{~K}$ smaller than for very thick films. Assuming a similar reduction in $T_{g}^{\prime}$ gives in our case (with $T_{\mathrm{g}} \approx 250 \mathrm{~K}$ ) $v_{0}^{\prime} \approx v_{0} / 1000$, so the segmental relaxation time $\tau^{\prime}=\lambda / v_{0}^{\prime}$ at the surface glass transition temperature $T_{g}^{\prime}$ would be of order $\sim 10$ s, i.e., similar to what is expected for glassy polymers at $T_{\mathrm{g}}$ (note: different glassy polymers exhibit very similar segmental relaxation times at $\left.T_{\mathrm{g}}\right)^{29}$

To summarize, it is assumed that the polymer segments at the rubber surface have higher mobility than in the bulk. Of course, if the polymer segments interact strongly with a substrate, it may have a lower mobility than in the bulk. However, we believe that because of short wavelength surface roughness and because of the finite sliding speed, the average surface separation (in the area of contact) at the sliding interface is relative large, giving rise to increased surface mobility of the polymer chains. The thickness of the surface layer with modified segment mobilities has been estimated for some polymers to be of order a few nanometers. ${ }^{16,17,30-34}$ This is also the surface region which most likely is of importance for the adhesive contribution to rubber friction, and it is therefore natural that we obtain a smaller activation energy for the adhesive contribution to rubber friction than that obtained for the bulk viscoelastic modulus.

We note here, however, that it is not clear that the temperature dependency of the adhesive contribution to rubber friction is determined by the rubber properties alone. The picture of rubber friction described above, involving rubber molecules or rather nanometer sized patches of

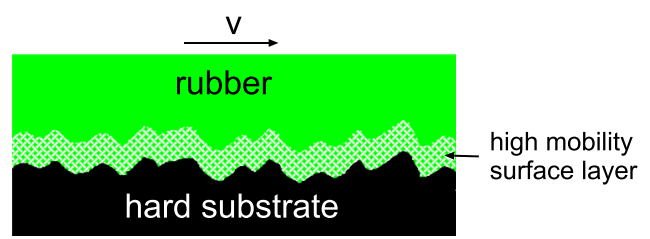

FIG. 5. Rubber block in sliding contact with a hard substrate with shortwavelength roughness. At the interface occurs a thin (a few nanometer) surface layer where the rubber polymer segments have higher mobility than in the bulk due to increased free volume. rubber at the rubber surface binding to the substrate in pinned domains, involves also energy barriers for breaking the substrate bonds. However, if these barriers are smaller than the barrier for segmental motion, they may give a weaker temperature dependency than that resulting from segmental motion.

\section{Shearing of thin transfer film}

At temperatures below the rubber glass transition temperature $T_{\mathrm{g}}$, rubber material exhibits stress-strain relations very similar to those of metals and some glassy polymers with a characteristic yield stress (see Sec. VI).

The friction coefficient we observe for rubber materials sliding on concrete at temperatures well below the glass transition temperature is $\mu \approx 0.5-0.6$ (see Fig. 2 and below). This is very similar to what we observe for glassy polymers on the same concrete surface. Thus in Fig. 6, we show the kinetic friction coefficient as a function of the sliding speed for polyethylene and PMMA on the concrete. The measurements were performed at $T=20{ }^{\circ} \mathrm{C}$ at low nominal contact pressure (about $10 \mathrm{kPa}$ ).

Several measurements have been performed of the shear stress when polymers are sliding on smooth surfaces. In particular, Whitten et al. ${ }^{35,36}$ studied the sliding of spherical glass balls on glassy polymers. In these experiments, most likely thin polymer films were transferred to the glass surfaces so the sliding contact was most likely the polymer on the same polymer. All the studied polymers exhibited similar frictional shear stress which increased linearly with the contact pressure: $\tau_{\mathrm{f}}=\tau_{0}+\beta p$. For example, for PMMA, the authors found $\tau_{0} \approx 30 \mathrm{MPa}$ and $\beta \approx 0.10$. If we assume that when PMMA is slid on the concrete surface a polymer transfer film is formed on the concrete surface and if we assume the PMMA penetration hardness $p_{\mathrm{Y}} \approx 100 \mathrm{MPa}$, we get $\tau_{\mathrm{f}} \approx 50 \mathrm{MPa}$ and the friction coefficient $\mu=\tau_{\mathrm{f}} / p_{\mathrm{Y}} \approx 0.5$, in good agreement with our experimental results.

We believe that for temperatures well below the glass transition temperature, rubber friction on hard and rough substrate surfaces is determined mainly by shearing of thin transfer films just as for the glassy polymers discussed above. That is, we expect transfer of rubber molecules to the concrete surface (see Fig. 21) and that shearing of the interface gives the observed

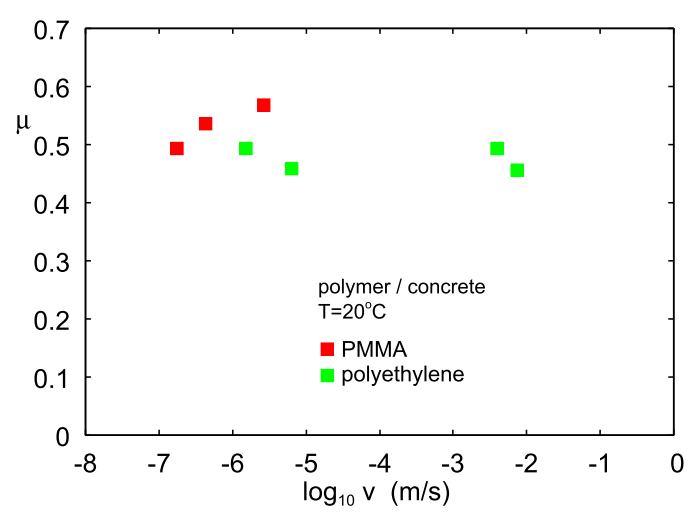

FIG. 6. The friction coefficient as a function of the sliding speed for polyethylene and PMMA on concrete. Measurements at $T=20^{\circ} \mathrm{C}$ at low nominal contact pressure (about $10 \mathrm{kPa}$ ). 
friction coefficients. We will discuss this topic in detail in Sec. VI.

\section{Construction of the friction master curve}

In his pioneering study, Grosch was able to construct smooth friction master curves for the friction coefficient by shifting the measured friction velocity segments $\mu(v)$, obtained at different temperatures, along the velocity axis using the bulk viscoelastic shift function $a_{T}$. We have found in earlier studies, and also in the present study (see below), that this is not the case for the systems we have studied. Grosch studied sliding friction on sand paper and for this substrate, very high wear rates occur while for more common surfaces such as concrete or asphalt road surfaces the wear rate is much smaller and this may be the reason for the different results.

We have found that the adhesive contribution to the friction coefficient has a different temperature dependency than the viscoelastic contribution (and the contact area) which depends on the bulk viscoelastic shift factor $a_{T}$. Hence it is not possible to construct master curves for the friction coefficient but only for the frictional shear stress. To obtain the frictional shear stress master curve we use (2) to obtain

$$
\tau_{\mathrm{f}}=p_{0} \frac{A_{0}}{A}\left(\mu-\mu_{\text {visc }}\right) .
$$

We calculate the viscoelastic contribution $\mu_{\mathrm{visc}}$ and the contact area $A$, which both depend on the temperature via the viscoelastic shift factor $a_{T}$, using the Persson theory. From the measured friction coefficient velocity segments $\mu(v)$, obtained at different temperatures, we obtain the shear stress velocity segments $\tau_{\mathrm{f}}(v)$ at different temperatures, which we shift to obtain a smooth master curve. The resulting shift factor $a_{T}^{\prime}$ is found to have the form (4) for low sliding speed (or high temperatures).

The friction coefficient master curve shown in Fig. 2 was obtained by shifting the high temperature velocity segments using the shift factor $a_{T}^{\prime}$ and the low temperature velocity segments using the bulk shift factor $a_{T}$. The reason this gave a relative smooth master curve is that in the velocity region where the adhesive contribution dominates, the viscoelastic contribution $\mu_{\mathrm{visc}}$ and the contact area $A$ are relatively weakly dependent on the sliding speed. Nevertheless, master curves for the friction coefficient, like the one shown in Fig. 2, are in general not accurate. Instead one should compare the theory results for the friction coefficient at every different temperature separately, as done in Figs. 12, 18, and 19.

In constructing friction master curves, one needs to measure the friction coefficient at such low sliding speeds that frictional heating can be neglected. For this reason, the highest sliding speed we use is $1 \mathrm{~mm} / \mathrm{s}$. In practical applications, frictional heating is of crucial importance and must be included in the theory analysis. ${ }^{37-39}$ Frictional heating occurs on many time and length scales and is a complex topic. Hence any fundamental approach to rubber friction must start with gaining a good understanding of rubber friction in the limiting case when frictional heating is unimportant and the temperature is the same everywhere in the rubber and substrate.
TABLE I. Summary of the glass transition temperatures of the SBR and the HNBR compounds. The glass transition temperature $T_{\mathrm{g}}$ is defined as the temperature where $\tan \delta(T)$ (for the frequency $\omega_{0}=0.01 \mathrm{~s}^{-1}$ ) is maximal.

\begin{tabular}{lcc}
\hline \hline Compound & $T_{\mathrm{g}}$ & Maximum of $\tan \delta$ \\
\hline SBR & $-44.0^{\circ} \mathrm{C}$ & 0.51 \\
HNBR-0 & $-23.0^{\circ} \mathrm{C}$ & 0.89 \\
HNBR-50 & $-23.3^{\circ} \mathrm{C}$ & 0.47 \\
\hline \hline
\end{tabular}

\section{EXPERIMENTAL}

We have studied the temperature and velocity dependency of rubber friction for three different rubber compounds on a concrete surface. Here we briefly describe the material properties and the experimental setup used for the friction experiments.

\section{A. Road surface}

The surface roughness of the concrete surface was studied using a stylus instrument and the surface roughness power spectrum $C(q)$ was calculated as described elsewhere ${ }^{40,41}$ and in Appendix B. The concrete surface has the rms roughness $\approx 0.12 \mathrm{~mm}$ and the surface roughness is well described as selfaffine fractal with the fractal dimension $D_{\mathrm{f}} \approx 2.1$.

\section{B. Rubber compounds}

The rubber compounds used were a SBR blend (tire tread compound) and filled and unfilled HNBR. The composition of the rubber compounds is given in Appendix B (see Tables II and III). Table I shows the rubber glass transition temperatures, here defined as the temperature where $\tan \delta(T)=\operatorname{Im} E(T) / \operatorname{Re} E(T)$ is maximal, where the viscoelastic modulus $E(T)$ is the (low strain) Young's modulus for the temperature $T$ and the frequency $\omega=0.01 \mathrm{~s}^{-1}$. The viscoelastic modulus master curves and the temperature-frequency shift factors $a_{T}$, as obtained for very low strain $(0.04 \%)$, are given for all the compounds in Appendix B. In Appendix B, we also present information about the non-linear stress-strain properties of all the rubber compounds, which are important for the calculation of the area of real contact.

\section{Linear friction tester}

We have developed a linear friction tester where the driving velocity can be specified. Figure 7 shows a schematic picture of the setup. The rubber is glued into the milling grove of the sample holder which is attached to the force cell (red block in the figure). The rubber specimen can move in the

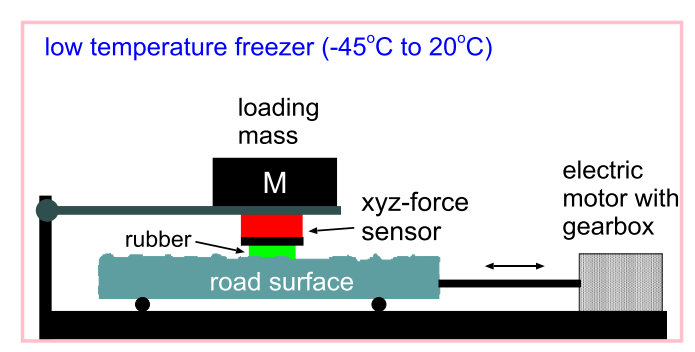

FIG. 7. Schematic picture of the low-temperature friction instrument allowing for linear reciprocal motion. 
vertical direction to adapt to the substrate profile. The normal load can be changed by adding additional steel plates on top of the force cell. The substrate sample is attached to the machine table which is moved by a servo drive via a gearbox in a translational manner. Here we control the relative velocity between the rubber specimen and the substrate sample while the force cell acquires information about normal force as well as friction force.

With the current configuration, it is possible to move the rubber specimen with velocities from $1 \mu \mathrm{m} / \mathrm{s}$ up to $1.25 \mathrm{~cm} / \mathrm{s}$. The temperature inside the deep freezer can be varied from $20{ }^{\circ} \mathrm{C}$ to $-45^{\circ} \mathrm{C}$. After the velocity profile measurement is finished, we change the temperature inside the deep freezer and repeat the whole velocity profile again.

The rubber samples used in this study were of two types: For the SBR compound, we cut out a rectangular rubber specimen (typical dimension is $3 \times 7 \mathrm{~cm}$ ) and glue it to the sample holder. We round off the sharp edges using a scalpel. For the HNBR compounds, we instead used half-cylinder shaped samples. The cylinders were $7 \mathrm{~cm}$ long and the radius was $1.5 \mathrm{~cm}$. We slid the cylinders in the direction orthogonal to the cylinder axis, but before the experiments we run in the samples (on a concrete surface) so that $\mathrm{a} \approx 10 \mathrm{~mm}$ wide nearly flat surface region was formed (see Fig. 32, which shows optical pictures of this surface area).

\section{EXPERIMENTAL RESULTS FOR THE SBR COMPOUND}

Figure 8 shows for the SBR compound the measured friction coefficient velocity segments, for temperatures between $T=-40{ }^{\circ} \mathrm{C}$ to $T=20{ }^{\circ} \mathrm{C}$. There are different procedures possible to construct a friction master curve from the measured velocity segments. The simplest procedure, which was used by Grosch, is to directly shift the measured velocity segments to obtain a smooth master curve. Grosch claimed that he could obtain smooth master curves this way and that the shift factor was the same as the Williams-Landel-Ferry (WLF)like shift factor obtained from the bulk viscoelastic modulus. However, we have already found in earlier studies that this is not the case, at least not for filled compounds on asphalt or concrete road tracks (Grosch focused mainly on unfilled compounds on sandpaper). Thus, Fig. 9 shows the master curve obtained using the bulk viscoelastic shift factor $a_{T}$. Clearly, the

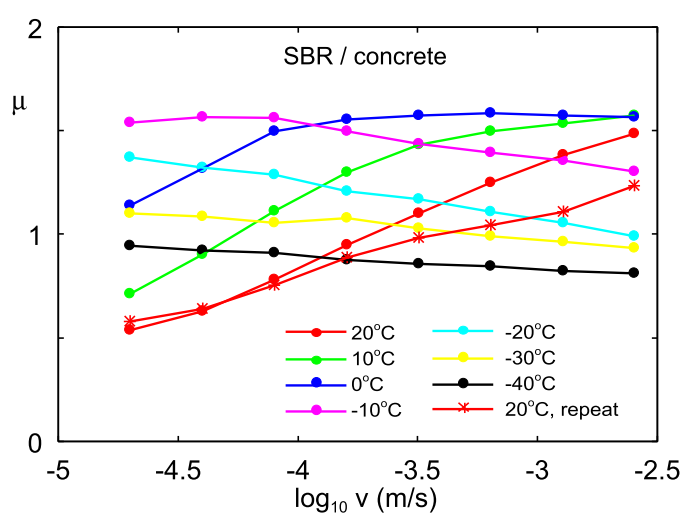

FIG. 8. Measured velocity segments $\mu(v)$, obtained at different temperatures.

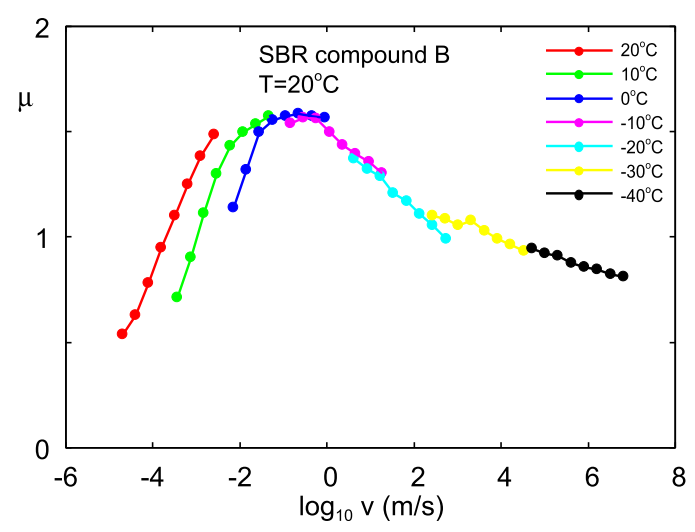

FIG. 9. Frictional master curve produced by shifting the measured velocity segments $\mu(v)$, obtained at different temperatures, to the reference temperature $T=20{ }^{\circ} \mathrm{C}$ using the bulk viscoelastic shift factor $a_{T}$ (green curve in Fig. 11).

adhesive contribution to the friction depends on the temperature in a different way than the viscoelastic contribution.

Figure 10 shows the friction coefficient master curve as obtained by shifting the $\mu(v)$ velocity segments in Fig. 8 using the shift factor $a_{T}^{\prime}$ shown in Fig. 11 (red stars). For low velocities (or high temperatures), where the adhesive contribution gives the dominating velocity dependency, the shifting procedure results in a shift factor as given by (4). However, as discussed in Sec. III, it is not strictly possible to shift the friction coefficient velocity segments directly to obtain a master curve since the shear stress $\tau_{\mathrm{f}}$ has a different temperature dependency than the viscoelastic contribution to the friction and also that of the contact area. Hence one should first calculate the shear stress velocity segments $\tau_{\mathrm{f}}(v)$ from the measured $\mu(v)$ via $\tau_{\mathrm{f}}(v)=p_{0}\left(A_{0} / A\right)\left(\mu(v)-\mu_{\mathrm{visc}}(v)\right)$. The different velocity segments $\tau_{\mathrm{f}}(v)$ obtained for the different temperatures can then be shifted to obtain a smooth master curve for $\tau_{\mathrm{f}}(v)$. In Appendix C, we show the frictional shear stress master curve obtained using this approach, and the shift factor $a_{T}^{\prime}$ is shown in Fig. 11 (red stars). The reason the same shift factor results in a relatively smooth master curve for the friction coefficient (see Fig. 10) is that the adhesive contribution occurs in a velocity region where the viscoelastic contribution and the contact area do vary relatively slowly with the sliding speed.

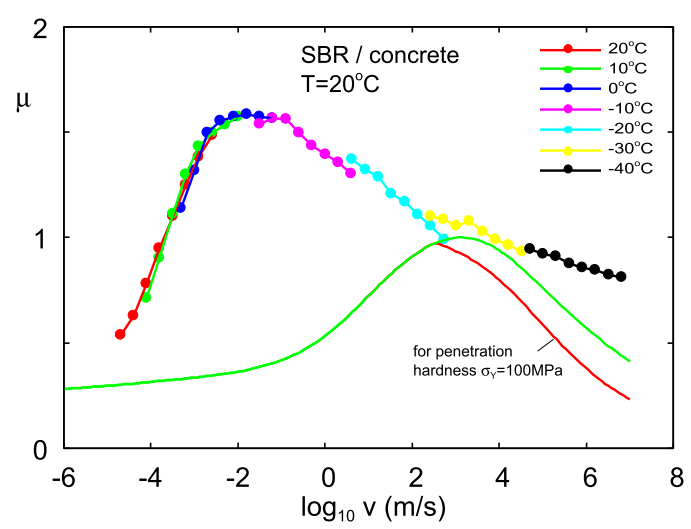

FIG. 10. Frictional master curve produced by shifting the measured velocity segments $\mu(v)$, obtained at different temperatures, to the reference temperature $T=20{ }^{\circ} \mathrm{C}$ using the shift factor $a_{T}^{\prime}$ shown in Fig. 11 (red stars). 


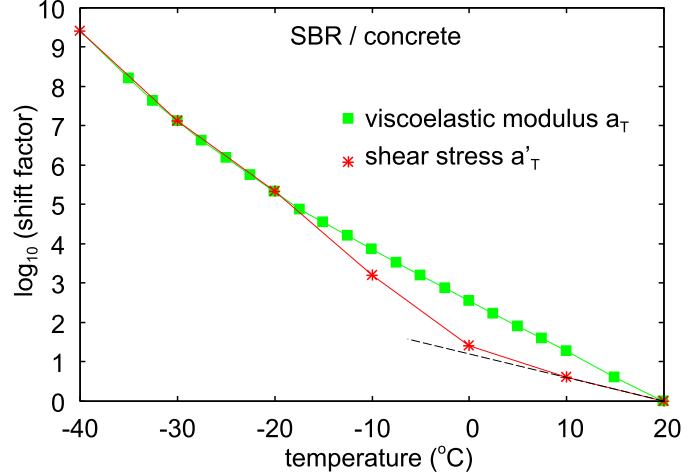

FIG. 11. Red stars: The shift factor $a_{T}^{\prime}$ used to obtain the master curve in Fig. 28. Green squares: the bulk viscoelastic shift factor $a_{T}$. The dashed line is the Arrhenius shift factor (4).

In Fig. 12, we show the friction coefficient as a function of sliding speed for several different temperatures $T=20,10,0,-10,-20,-30$, and $-40{ }^{\circ} \mathrm{C}$. The blue squares
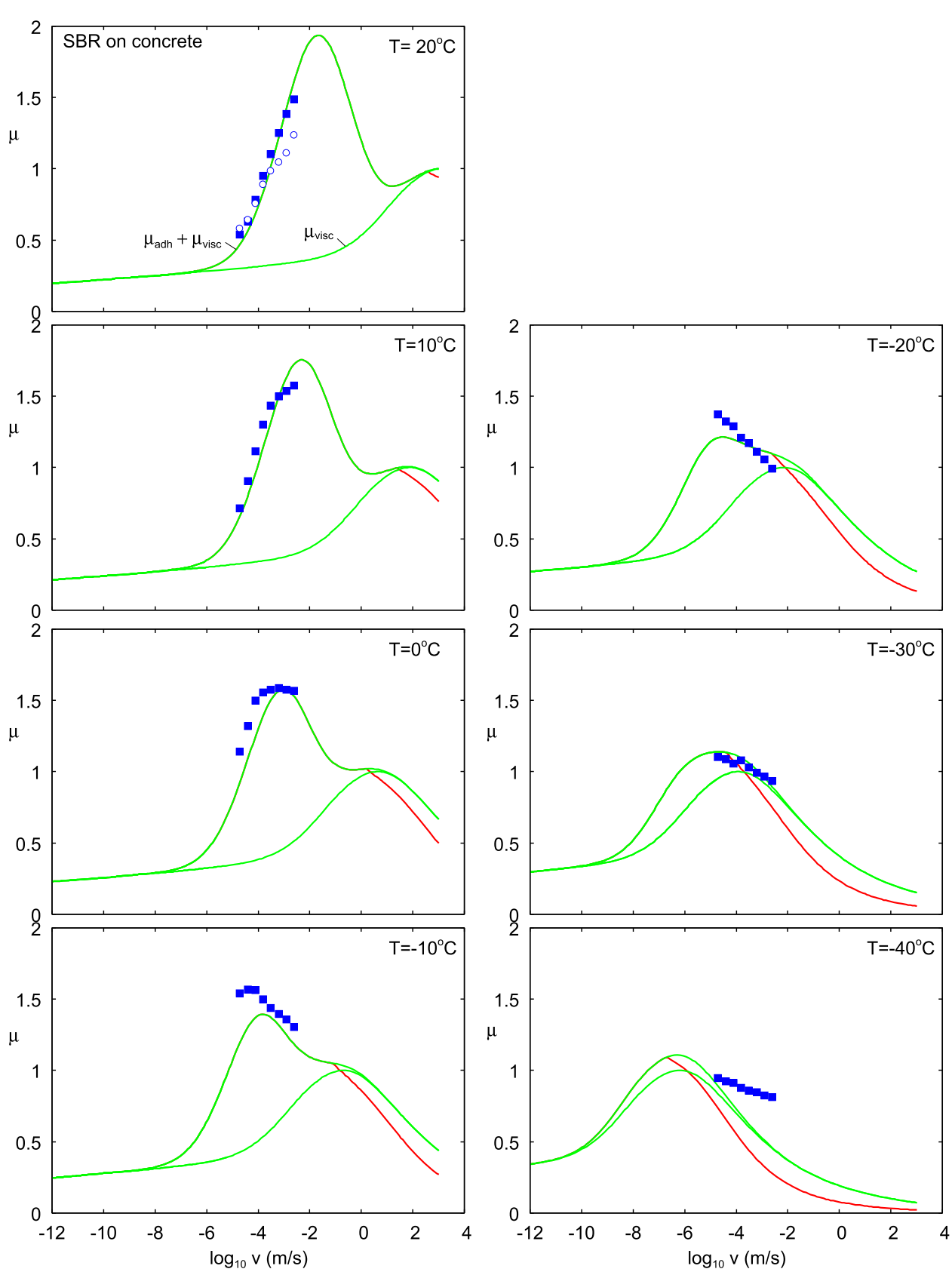

are the measured data and the green lines the calculated friction coefficient including only the viscoelastic contribution $\mu_{\text {visc }}$ (lower green curves) and the viscoelastic plus adhesive contribution $\left(\mu_{\mathrm{visc}}+\mu_{\mathrm{ad}}\right)$ (upper green curve). In the calculation, we have not included the contribution from shearing the transfer film, which we believe is important for low temperatures. The adhesive contribution is assumed to have the temperature dependency given by the Arrhenius law (4). The red curves are the results obtained by including the finite penetration hardness in the calculations, while the green curves are without plastic yielding.

\section{EXPERIMENTAL RESULTS FOR THE FILLED AND UNFILLED HNBR COMPOUNDS}

The SBR compound studied above has the glass transition temperature $T_{\mathrm{g}} \approx-44{ }^{\circ} \mathrm{C}$ which is much lower than for the unfilled and filled HNBR compounds we study in this
FIG. 12. The friction coefficient for the SBR compound as a function of sliding speed for several different temperatures $T=20,10,0,-10,-20,-30$, and $-40{ }^{\circ} \mathrm{C}$. Blue squares: The measurements start at $T=20^{\circ} \mathrm{C}$ and end with the measurement at $T=-40{ }^{\circ} \mathrm{C}$. Open circles: After the measurement at $T=-40{ }^{\circ} \mathrm{C}$, the temperature is returned to $T=20^{\circ} \mathrm{C}$ and a new measurement is performed to check how much the friction has changed due to contamination of the sliding surfaces. The green lines are theory predictions discussed in the text. 


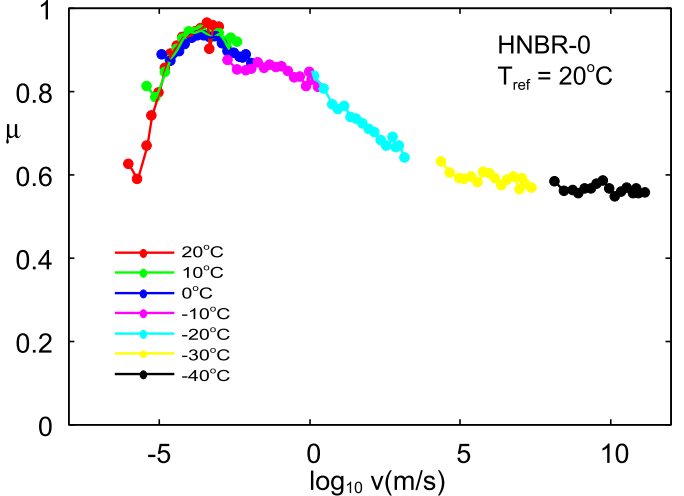

FIG. 13. The friction coefficient master curve at $T=20^{\circ} \mathrm{C}$ for the HNBR0 compound obtained using the shift factors $a_{T}^{\prime}$ shown in Fig. 14 (red stars).

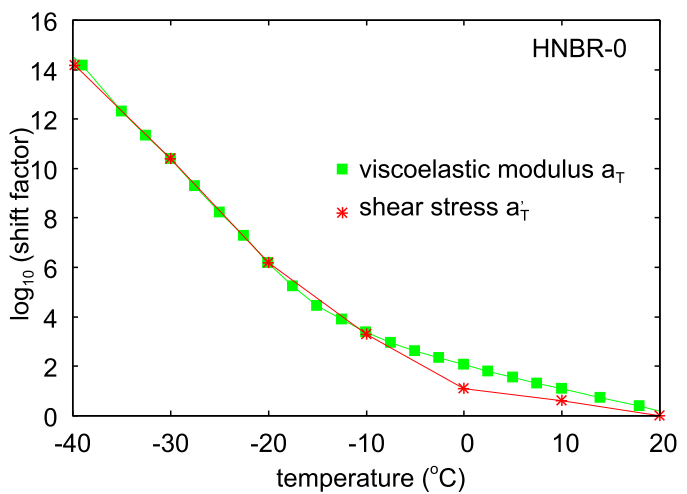

FIG. 14. Red stars: The shift factor $a_{T}^{\prime}$ used to obtain the master curve in Fig. 29. Green squares: the bulk viscoelastic shift factor $a_{T}$.

section where $T_{\mathrm{g}} \approx-23{ }^{\circ} \mathrm{C}$. This implies that the viscoelastic and adhesive contributions to the rubber friction for the HNBR compounds become unimportant at much higher temperatures (and hence lower sliding speeds) than for the SBR compound.

In Fig. 13, we show the friction coefficient master curve at $T=20^{\circ} \mathrm{C}$ for the HNBR- 0 compound obtained using the shift factors $a_{T}^{\prime}$ shown in Fig. 14 (red stars). In Figs. 15 and 16, we show the corresponding results for the HNBR-50 compound. The shift factors $a_{T}^{\prime}$ in Fig. 14 and 16 are obtained by shifting the shear stress velocity segments, $\tau_{\mathrm{f}}(v)$, obtained at different

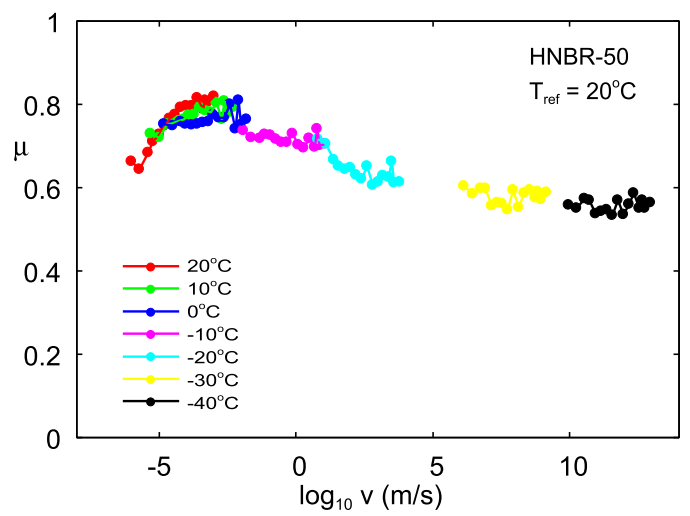

FIG. 15. The friction coefficient master curve at $T=20{ }^{\circ} \mathrm{C}$ for the HNBR50 compound obtained using the shift factors $a_{T}^{\prime}$ shown in Fig. 16 (red stars).

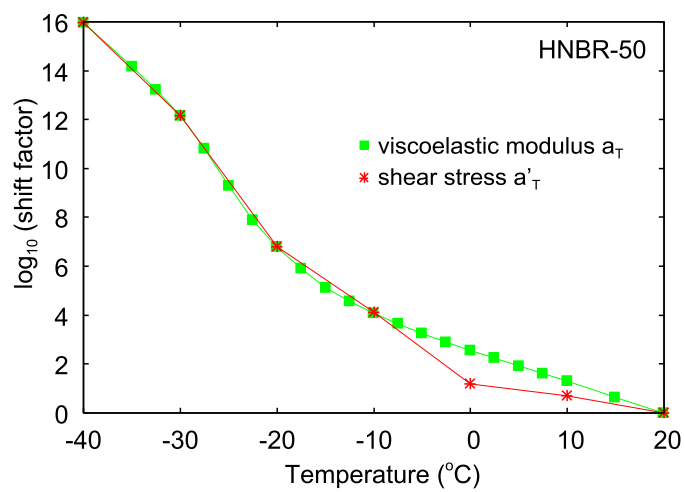

FIG. 16. Red stars: The shift factor $a_{T}^{\prime}$ used to obtain the master curve in Fig. 30. Green squares: the bulk viscoelastic shift factor $a_{T}$.

temperatures, in order to obtain smooth master curves for $\tau_{\mathrm{f}}(v)$ (see Appendix C).

In Fig. 17, we compare the friction coefficient master curve for the HNBR-0 (red curve) and HNBR-50 (green curve) compounds. It is interesting to note that for the lowest 2 temperatures, the friction is the same for the filled and unfilled compounds. For these temperatures, we believe that the friction arises from shearing a thin transfer film.

Figsures 18 and 19 show the measured friction coefficients (blue squares) and the theory predictions (green lines), including only the viscoelastic contribution (lower green lines) and the viscoelastic plus adhesive contribution (upper green lines), where for the latter we have used the shift factor $a_{T}^{\prime}$ given by (4). In the calculation, we have not included the contribution from shearing the transfer film. Clearly for both the filled and unfilled HNBR compounds for the two lowest temperatures, the adhesive and viscoelastic contributions are very small and shearing the transfer film dominates the friction.

\section{RUBBER FRICTION AT LOW TEMPERATURES}

At temperatures below the glass transition temperature $T_{\mathrm{g}}$, rubber material exhibits stress-strain relations very similar to those of metals with a characteristic yield stress. This is illustrated in Fig. 20 which shows the stress-strain relation at $T \approx-40{ }^{\circ} \mathrm{C}$ for the unfilled and filled HNBR

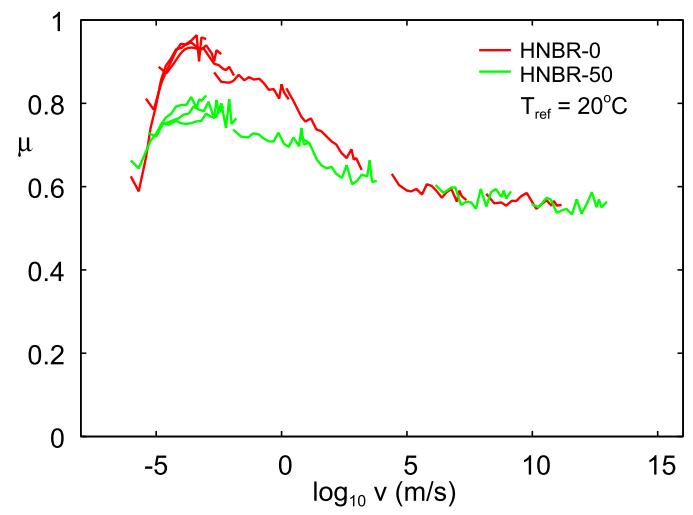

FIG. 17. The friction coefficient master curve at $T=20{ }^{\circ} \mathrm{C}$ for HNBR-0 (red curve) and HNBR-50 (green curve) compounds obtained using the shift factors $a_{T}^{\prime}$ shown in Figs. 14 and 16 (red stars), respectively. 

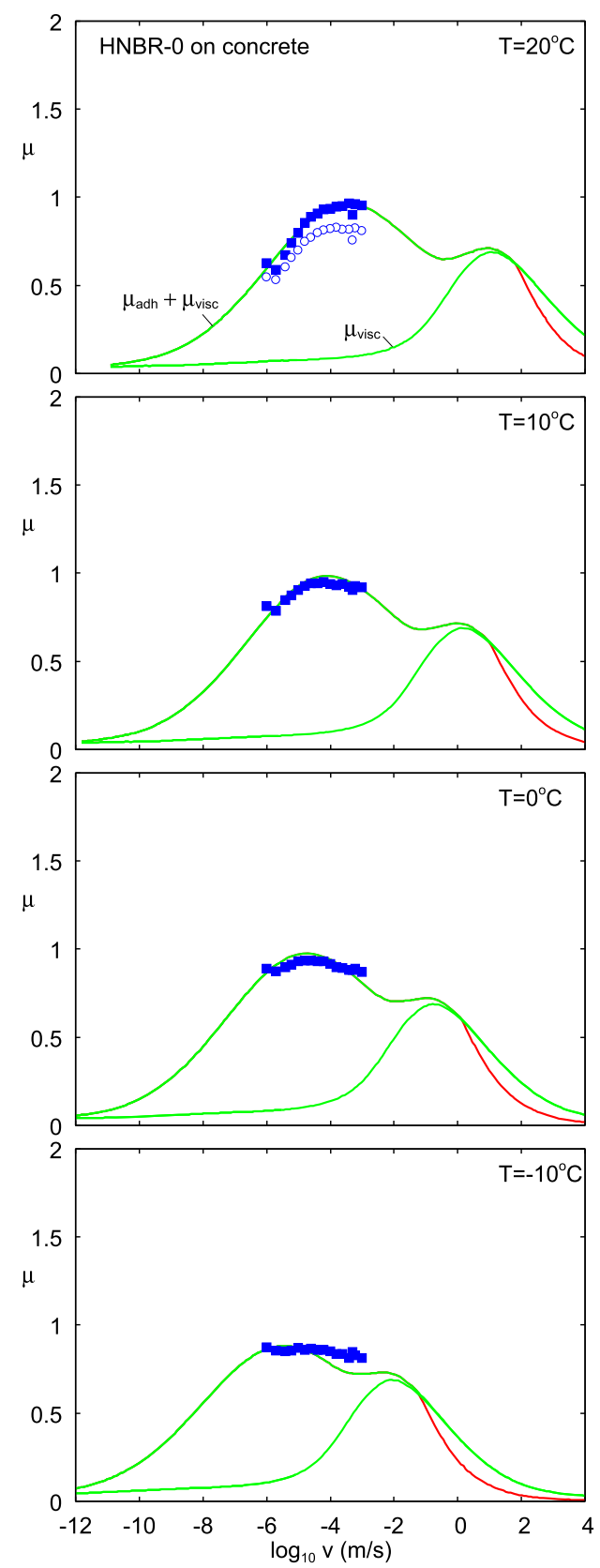
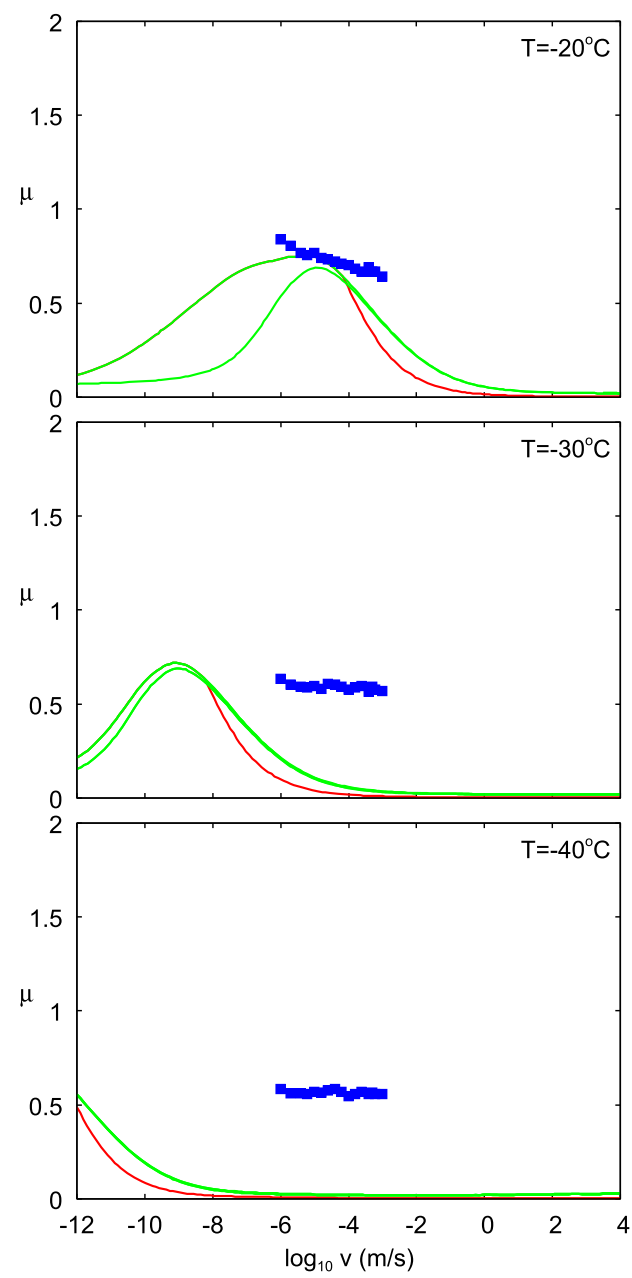

FIG. 18. The friction coefficient for the unfilled HNBR compound as a function of sliding speed for several different temperatures $T=20,10,0,-10,-20$, -30 , and $-40{ }^{\circ} \mathrm{C}$. Blue squares: The measurements start at $T=20^{\circ} \mathrm{C}$ and end with the measurement at $T=-40^{\circ} \mathrm{C}$. Open circles: After the measurement at $T=-40^{\circ} \mathrm{C}$, the temperature is returned to $T=20^{\circ} \mathrm{C}$ and a new measurement is performed to check how much the friction has changed due to contamination of the sliding surfaces. The green lines are theory predictions discussed in the text. compounds. The initial slope of the $\sigma(\epsilon)$ curves corresponds to the Young's modulus $E \approx 2.6 \mathrm{GPa}$ and $2.0 \mathrm{GPa}$ for the filled and unfilled compounds, respectively, but at the strain $\epsilon \approx 0.02$ the material yields plastically. For both compounds, the yield stress (in elongation) is about $40 \mathrm{MPa}$. We note, however, that when the temperature is increased above $T_{\mathrm{g}}$, the rubber samples nearly fully recover their original shape, i.e., most of the deformations disappear. Hence, the plastic deformations of rubber at low temperatures do not involve breaking of (strong) covalent bonds (e.g., hydrocarbon or sulfur bonds), but rather involve changes in the arrangement of the polymer chains. This involves segments of the polymer chains moving over energetic barriers during the plastic deformations, which at temperatures $T<T_{\mathrm{g}}$ are too large to be overcome by thermal fluctuations during time periods of practical importance. However, for temperatures $T>T_{\mathrm{g}}$, the thermal fluctuations are large enough to move the segments over the energy barriers so the rubber returns to its original free energy minimum state.

The friction coefficient we observe for rubber materials at temperatures well below the glass transition temperature is $\mu \approx 0.5-0.6$ (see Figs. 18 and 19 ). As shown in Sec. II, this is very similar to what we observe for glassy polymers sliding on the same concrete surface (see Fig. 6). When a glassy polymer, at temperatures below the glass transition temperature, is sliding on a hard rough substrate surface, plastic deformation and plowing typically occur. Plowing is caused by asperities of a hard material penetrating into a softer material and plowing out a groove by a plastic flow in the softer material. We believe that for temperatures well below the glass transition temperature, rubber friction on hard and randomly rough substrate surfaces involves plastic deformation, plowing, and wear particle production. However, we show in Appendix D that these processes do not give the main contribution to the observed 

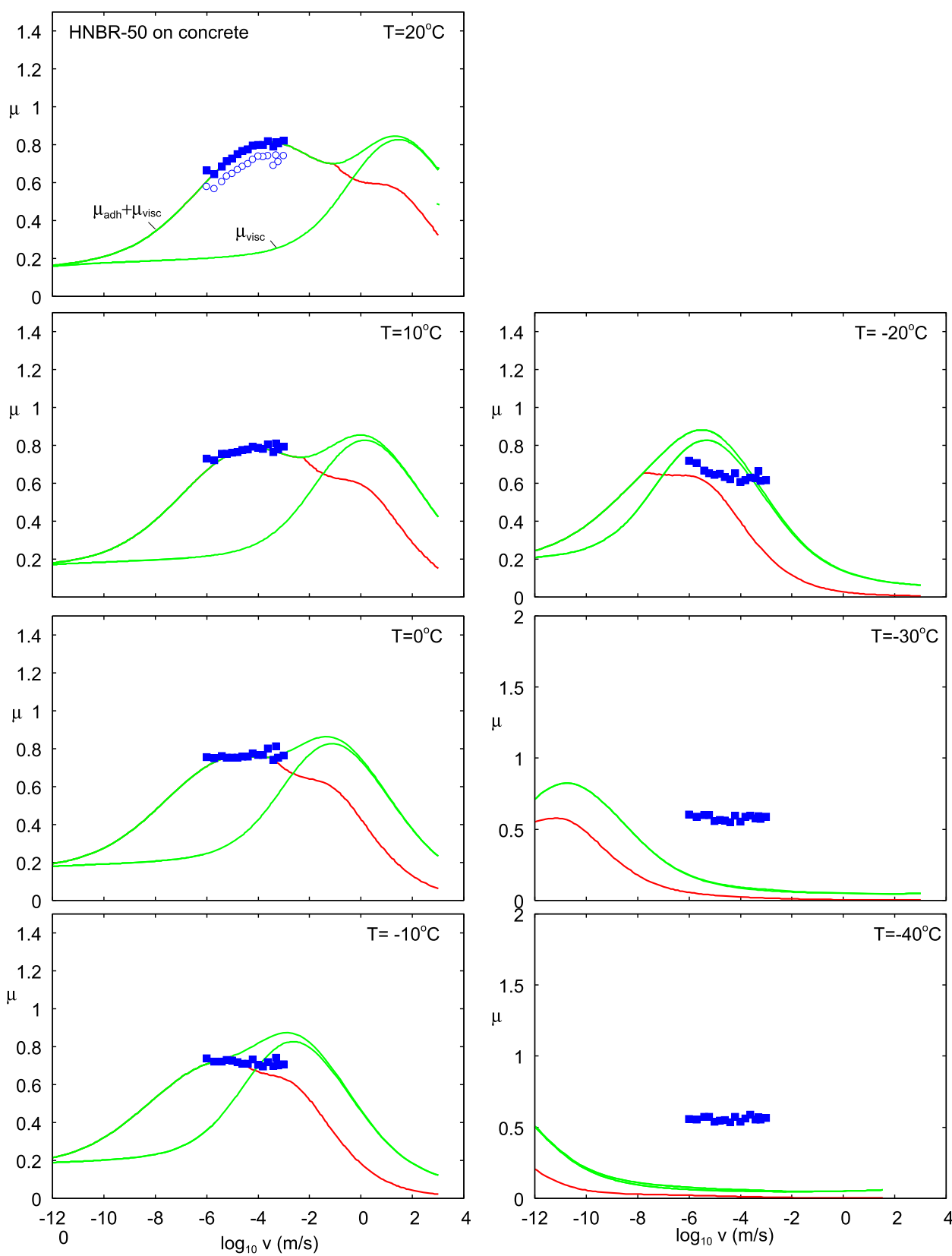

FIG. 19. The friction coefficient for the filled HNBR compound as a function of sliding speed for several different temperatures $T=20,10,0,-10,-20,-30$, and $-40{ }^{\circ} \mathrm{C}$. Blue squares: The measurements start at $T=20^{\circ} \mathrm{C}$ and end with the measurement at $T=-40{ }^{\circ} \mathrm{C}$. Open circles: After the measurement at $T=-40{ }^{\circ} \mathrm{C}$, the temperature is returned to $T=20^{\circ} \mathrm{C}$ and a new measurement is performed to check how much the friction has changed due to contamination of the sliding surfaces. The green lines are theory predictions discussed in the text. friction coefficient, which instead is due to shearing of a transfer film deposited on the concrete surface by the rubber sliding action (see Fig. 21).

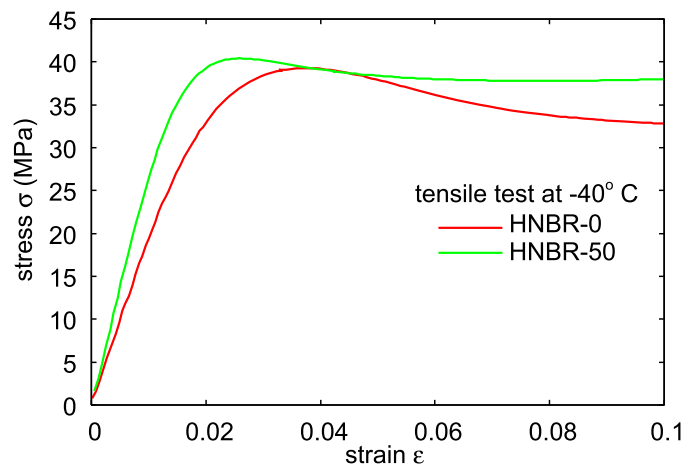

FIG. 20. The tensile stress-strain curves of unfilled and filled HNBR at $T=-40{ }^{\circ} \mathrm{C}$.
Rubber friction in the low temperature region is similar to the friction when a hydrocarbon slab is slid on another hydrocarbon slab of the same material. For polymer sliding on the same polymer experiments, ${ }^{35}$ and computer simulations, ${ }^{36}$ give shear stresses which increase linearly with the contact pressure, and which typically equal to $\approx 50 \mathrm{MPa}$ at the contact pressure $\approx 100 \mathrm{MPa}$; this corresponds to a friction coefficient $\mu \approx 0.5$ as observed in our experiments. This picture requires that there is a transfer of a nanometer (or more) film of rubber hydrocarbon molecules to the concrete surface in the contact region, as indeed observed (see Fig. 21), and that the two solids slip at the interface between the adsorbed hydrocarbon film and the rubber surface. For this situation, the relative high friction observed in the computer simulations results from the fact that the atomic corrugation on the two surfaces is the same which allows locally (in space and time) commensurate-like regions to form. 


\section{DISCUSSION}

We have found in this paper, and in an earlier study, that the adhesive contribution to the rubber friction obeys a different temperature dependency than that associated with the bulk viscoelastic modulus. We attribute this to a smaller activation barrier for segmental motion of the rubber hydrocarbon chains at the surface than in the bulk. Thus, in a typical case, the activation barrier for segmental motion is $\approx 2 \mathrm{eV}$ in the bulk and $\approx 1 \mathrm{eV}$ at the surface (or rather the interface). For the rubberair interface, this is indeed expected based on standard freevolume arguments and is also found in experiments. For the contact between rubber and a flat surface, the situation is less clear, but we believe it holds also for the concrete surface we use, and most other surfaces of practical interest, because of the large surface roughness prevailing on most cases (see Fig. 5).

For temperatures below the glass transition temperature, or equivalently, for very high sliding speeds (neglecting frictional heating), there is another contribution to the frictional shear stress which we have suggested to result from shearing of a transfer film. However, from a practical point of view, this is not so important as frictional heating will result in rubber temperatures well above the glass transition temperature in most applications such as for tires. Thus, in most cases, the friction can be obtained as the sum of the viscoelastic contribution and the adhesive contribution.

In the context of F1 racing, it has been observed that transfer of rubber (or rubber derived wear products) from racing tires to the road surface can increase the friction. This may be due to a smoothing of the road asperities at short length scales, which increases the tire-road contact area and the adhesive contribution to the friction. At the same time, the viscoelastic contribution to the friction from the surface roughness is reduced, but for racing tires the adhesive contribution may be the most important.

It has been observed that if a road surface is run-in for a particular type of tires, it can result in very low friction for other tires produced using different rubber compounds. This observation is consistent with the picture of the adhesion contribution to rubber friction presented above. Thus, at high enough temperature, polymer chains on one surface can easily interdiffuse with the polymer chains on an opposite surface (here the road surface contaminated by rubber wear products) if the polymers on the two surfaces are identical. If the polymers are different, interdiffusion is less likely and a "phase-separated" weakly interacting interface may prevail. This would result in an adhesion contribution to the friction which may be much smaller in the latter case as compared to the former case. And even if interdiffusion does not occur (as would be the case at low temperature), the same polymer on both sides results in a more commensurate-like contact with higher barriers for lateral motion than when the polymers differ.

The influence of interdiffusion (or commensurability) on friction is well known and general: to obtain low friction between two glassy polymers in mechanical contact, one should use different polymers. As an example, ${ }^{36}$ for polyoxymethylene sliding on polyoxymethylene or for polyethylene on polyethylene, the friction coefficient (at the velocity $1 \mathrm{~mm} / \mathrm{s})\left(\mu_{\mathrm{k}} \approx 0.55\right)$ is about 5 higher than for polyoxymethylene on polyethylene $\left(\mu_{\mathrm{k}} \approx 0.1\right)$. In these applications, the polymers are typically used at room temperature, i.e., well below the glass transition temperatures and one may think that the chain mobility is too low to allow for interdiffusion. However, in the sliding contact, the local temperature in the asperity contact regions may be so high as to increase the chain mobility to the point where chain interdiffusion would occur. In fact, in some cases, even local melting of the polymers has been observed. In addition, as pointed out before, at the surface (here interface), the mobility of the polymer chains will be higher than in the bulk (i.e., the local glass transition at the surface is lower than in the bulk) and this will reduce the barrier for interdiffusion. And even if no interdiffusion can occur due to low chain mobility, the commensurability argument above shows that one expects higher friction when using the same polymer materials on both sides.

\section{SUMMARY AND CONCLUSION}

In this paper, we have presented experimental results obtained at different sliding speeds and temperatures for two chemically different rubbers compounds, namely, SBR and HNBR (filled and unfilled) and we found the following:

(1) The temperature dependency of the shear stress $\tau_{\mathrm{f}}$, for temperatures above the rubber glass transition temperature $T_{\mathrm{g}}$, is weaker than that of the bulk viscoelastic modulus. We propose that the origin of the temperature dependency of $\tau_{\mathrm{f}}$ for $T>T_{\mathrm{g}}$ is determined by the rubber molecule segment mobility at the sliding interface, which is higher than in the bulk due to increased freevolume effect due to short-wavelength surface roughness. We note that the sliding movement may "generate" free volume by "pulling" on polymer strands (the sequences between cross-linked points in the rubber), which cannot relax completely during the time available in the course of the sliding movement. ${ }^{48}$

(2) For temperatures $T<T_{\mathrm{g}}$, the shear stress $\tau_{\mathrm{f}}$ is nearly velocity independent and of similar magnitude as observed for glassy polymers such as PMMA or polyethylene. In this case, the rubber undergoes plastic deformations in the asperity contact regions and the frictional shear stress is due to slip at the interface between the rubber and a hydrocarbon film adsorbed on the concrete surface. The hydrocarbon film (of nanometer thickness or more) is assumed to result from the transfer of molecules from the rubber to the substrate surface.

\section{ACKNOWLEDGMENTS}

This work was supported in part by the Research Council of Norway (Project No. 234115 in the Petromaks2 programme). This work was performed within a Reinhart-Koselleck project funded by the Deutsche Forschungsgemeinschaft (DFG). We would like to thank DFG for the project support under the reference German Research Foundation Grant No. DFG-grant:MU 1225/36-1. 
The research work was also supported by Grant Nos. DFGgrant:PE 807/10-1 and DFG-grant:HE 4466/34-1. We thank Anton Akulichev for providing tensile test data at low temperatures and Wim Pyckhout-Hintzen for allowing us to use his DMA instrument for the measurements of the rubber viscoelastic modulus.

\section{APPENDIX A: VISCOELASTIC CONTRIBUTION TO RUBBER FRICTION}

At present, two different theories have been used to calculate the area of real contact and the viscoelastic contribution to rubber friction. Here we consider the simplest case of sliding at a constant speed and at such low speed that frictional heating can be neglected.

In the theory of Persson, the friction force acting on a rubber block squeezed with the stress $p_{0}$ against a hard randomly rough surface is given by $9,10,15,37$

$$
\begin{aligned}
\mu_{\mathrm{visc}} \approx & \frac{1}{2} \int_{q_{0}}^{q_{1}} d q q^{3} C(q) S(q) P(q) \\
& \times \int_{0}^{2 \pi} d \phi \cos \phi \operatorname{Im} \frac{E\left(q v(t) \cos \phi, T_{0}\right)}{\left(1-v^{2}\right) p_{0}},
\end{aligned}
$$

where $T_{0}$ denotes the temperature and where

$$
P(q)=\frac{1}{\sqrt{ } \pi} \int_{0}^{\sqrt{ } G} d x e^{-x^{2} / 4}=\operatorname{erf}\left(\frac{1}{2 \sqrt{ } G}\right)
$$

where

$$
G(q)=\frac{1}{8} \int_{q_{0}}^{q} d q q^{3} C(q) \int_{0}^{2 \pi} d \phi\left|\frac{E\left(q v \cos \phi, T_{0}\right)}{\left(1-v^{2}\right) p_{0}}\right|^{2} .
$$

Note that $P(q)=A(q) / A_{0}$ is the (normalized) contact area observed at the magnification $\zeta=q / q_{0}$ (see Sec. II).

The factor $S(q)$ in (A1) is a correction factor which takes into account that the asperity-induced deformations of the rubber are smaller as in the case if complete contact would occur in the (apparent) contact areas observed at the magnification $\zeta=q / q_{0}$. For contact between elastic solids, this factor reduces the elastic asperity-induced deformation energy and including this factor gives a distribution of interfacial separation in good agreement with experiments and exact numerical studies. ${ }^{47}$ The interfacial separation describes how an elastic (or viscoelastic) solid deforms and penetrates into the roughness valleys, and it is stressed here that these (time-dependent) deformations cause the viscoelastic contribution to rubber friction. We assume that the same $S(q)$ reduction factor as found for elastic contact is valid also for sliding contact involving viscoelastic solids. For elastic solids, it has been found that $S(q)$ is well approximated by

$$
S(q)=\gamma+(1-\gamma) P^{2}(q),
$$

where $\gamma \approx 1 / 2$ and here we use the same expression for viscoelastic solids, being in nature a geometrical parameter. Note that $S \rightarrow 1$ as $P \rightarrow 1$ which is an exact result for complete contact. In fact, for complete contact, the expression (A1) is exact.

The more general case of non-uniform sliding and including frictional heating was studied in Ref. 15.
In the theory of rubber friction, ${ }^{6,9}$ the viscoelastic contribution to the friction depends on the surface roughness power spectrum $C(q)$, where $q=2 \pi / \lambda$ is the wavenumber of a particular frequency component (with wavelength $\lambda$ ) of the roughness profile. Most surfaces have a self-affine fractal-like topography, where $C(q) \sim q^{-2(1+H)}$. Here the Hurst exponent typically is in the range $0.7<H<1$, corresponding to a fractal dimension $D_{\mathrm{f}}=3-H$ between 2 and 2.3 (see Refs. 19 and 40). Most surfaces have a roll-off region for $q<q_{\mathrm{r}}$, where $C(q)$ is approximately constant. In calculating the rubber friction, we include all the roughness components with wavenumber $q_{0}<q<q_{1}$. Here $q_{0}=2 \pi / L$, where $L$ is the linear size of the rubber block in the sliding direction. If $q_{0}<q_{\mathrm{r}}$, as is usually the case, the contact area and the viscoelastic contribution to the friction are nearly independent of $q_{0}$. We define the magnification $\zeta=q / q_{0}$. When we study the interface at the magnification $\zeta$, we do not observe roughness components with wavenumber $q>q_{0} \zeta$, and physical quantities observed at this magnification will therefore depend on the magnification.

We note that when calculating the viscoelastic contribution to rubber friction (and the contact area), it is necessary to introduce a large wavenumber cutoff $q_{1}=2 \pi / \lambda_{1}$, where $\lambda_{1}$ is the shortest surface roughness wavelength included in the contact mechanics calculation. For smooth surfaces, $\lambda_{1}$ may be of order atomic distances, or the average distance between cross-links, i.e., $q_{1} \approx 10^{9}-10^{10} \mathrm{~m}^{-1}$. For very rough surfaces like road surfaces, the cutoff may be related to the onset of rubber bond-breaking and wear processes, which appear in the contact regions at high enough magnifications as a result of large stresses and high temperatures. For road surfaces, this typically gives the cutoff $q_{1} \sim 10^{6}-10^{7} \mathrm{~m}^{-1}$.

\section{APPENDIX B: MATERIAL PROPERTIES}

The experiments reported above were performed on three rubber compounds sliding on a concrete surface. The composition of the rubber compounds is given in Tables II and III. Here we briefly describe the material properties, namely, the viscoelastic modulus of the rubber compounds and the surface roughness of the concrete surface.

\section{Surface roughness power spectrum}

As substrates for the rubber friction study, we use concrete surfaces. The concrete blocks have been used in several earlier studies. They are very stable (negligible wear).

TABLE II. Components of the HNBR compounds in parts per hundred rubber (phr) of the total compound.

\begin{tabular}{lcc}
\hline \hline Materials & HNBR-0 & HNBR-50 \\
\hline HNBR & 100 & 100 \\
Antioxidant & 3 & 3 \\
Stearic acid & 0.5 & 0.5 \\
Zinc oxide & 5 & 5 \\
Magnesium oxide & 10 & 10 \\
Plasticizers & 20 & 20 \\
Carbon black (N-330) & 0 & 50 \\
Peroxide & 10 & 10 \\
\hline \hline
\end{tabular}


TABLE III. Components of the SBR compound in volume $\%$ of the total compound.

\begin{tabular}{lc}
\hline \hline Materials & SBR \\
\hline Carbon black & 17.6 \\
Silica & 4.75 \\
Oil & 22.55 \\
Resin & 0.58 \\
\hline \hline
\end{tabular}

Figure 21 shows the concrete surface where the dark region arose from transfer of rubber material to the concrete surface during sliding at $v=1 \mathrm{~mm} / \mathrm{s}$ for two cycles (each cycle is $40 \mathrm{~cm}$ ).

The surface roughness of the concrete surface was studied using a stylus instrument. Figure 22 shows the surface roughness power spectrum of the concrete surface used in this study. The large wavenumber cutoff $q_{1}=8.1 \times 10^{6} \mathrm{~m}^{-1}$ or cut-off length $\lambda_{1}=2 \pi / q_{1} \approx 1 \mu \mathrm{m}$. The root-mean-square slope of the surface including all the roughness with wavenumber below $q_{1}$ is 1.3 .

\section{Viscoelastic modulus}

For rubber friction, it is necessary to have information about the complex elastic modulus over a rather large frequency range as well as at different strain values including very large strain of order $100 \%$. A standard way of measuring the viscoelastic modulus is to oscillatory deform the rubber sample with a constant strain or stress amplitude. This is done at different frequencies and then repeated at different temperatures. The results measured at different temperatures can be time-temperature shifted to a master curve at a chosen reference temperature covering a broad range of frequencies. This has been shown for unfilled polymers and is approximately true for filled rubber as well.

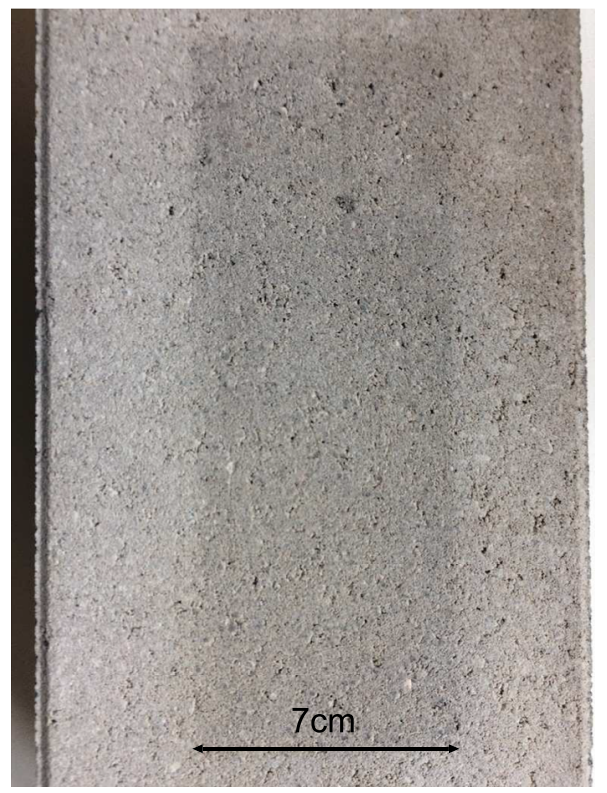

FIG. 21. The concrete surface used in the experiments. The dark region arose from transfer of rubber material (carbon black filled HNBR-50) to the concrete surface during sliding at $v=1 \mathrm{~mm} / \mathrm{s}$ for two cycles (each cycle is $40 \mathrm{~cm}$ ) at $T=-40{ }^{\circ} \mathrm{C}$.

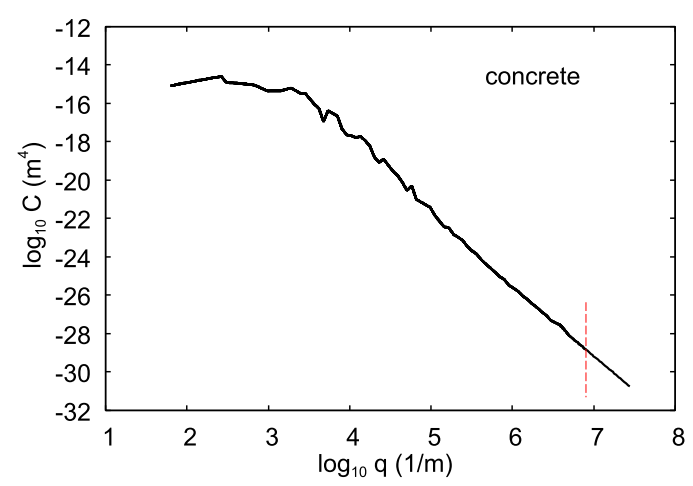

FIG. 22. The surface roughness power spectrum of the concrete surface used in this study. The large wavenumber cutoff $q_{1}=8.1 \times 10^{6} \mathrm{~m}^{-1}$ or cut-off length $\lambda_{1}=2 \pi / q_{1} \approx 1 \mu \mathrm{m}$.

In this section, we present results for the viscoelastic properties for SBR (tire tread compound) and for filled and unfilled HNBR rubbers. The HNBR with 36 wt. \% of acrylonitrile (ACN) and 96\% saturation of the butadiene groups was used in this study. The HNBR-50 compound contains carbon black filler and was prepared by a two-step procedure. In the first step, HNBR was compounded with curing agents, antioxidants, magnesium oxide, and plasticizers in an internal mixer to form a premix of HNBR. The second step involved mixing of the premix with peroxide in a two roll mill.

For our dynamic mechanical analysis (DMA) measurements, we use a Q800 Dynamic Mechanical Analysis (DMA) instrument produced by TA Instruments. The machine is run
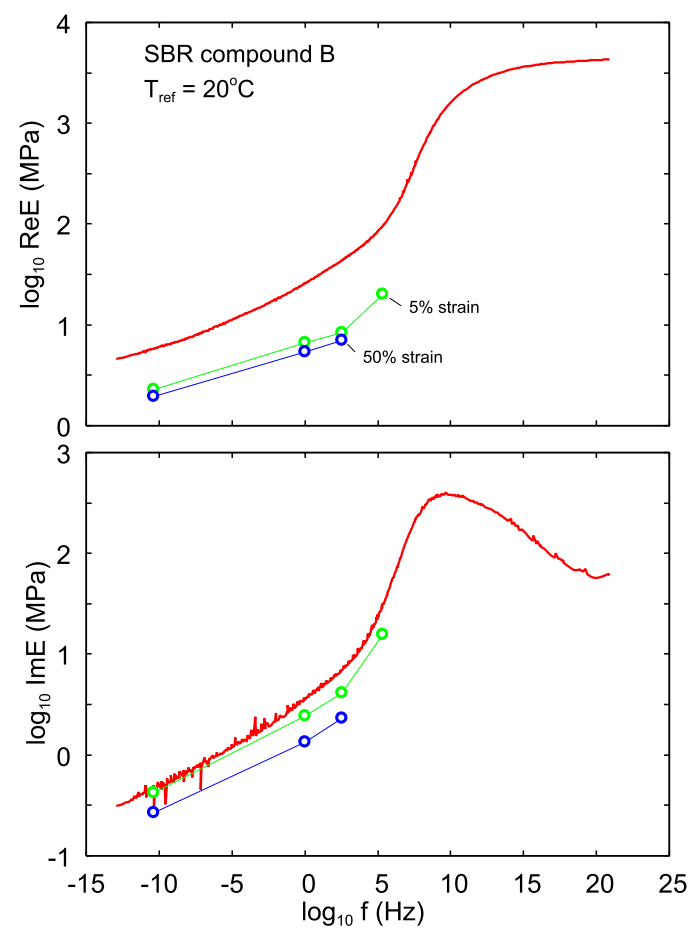

FIG. 23. Thick lines: The viscoelastic modulus master curve for the SBR compound obtained at very low strain (linear response region), $0.04 \%$ strain. Circles: the effective modulus at large strain as obtained for the frequency $f=1 \mathrm{~Hz}$ at different temperatures and then shifted along the frequency axis to the reference temperature $20^{\circ} \mathrm{C}$ using the (low-strain) viscoelastic shift factor $a_{T}$. 

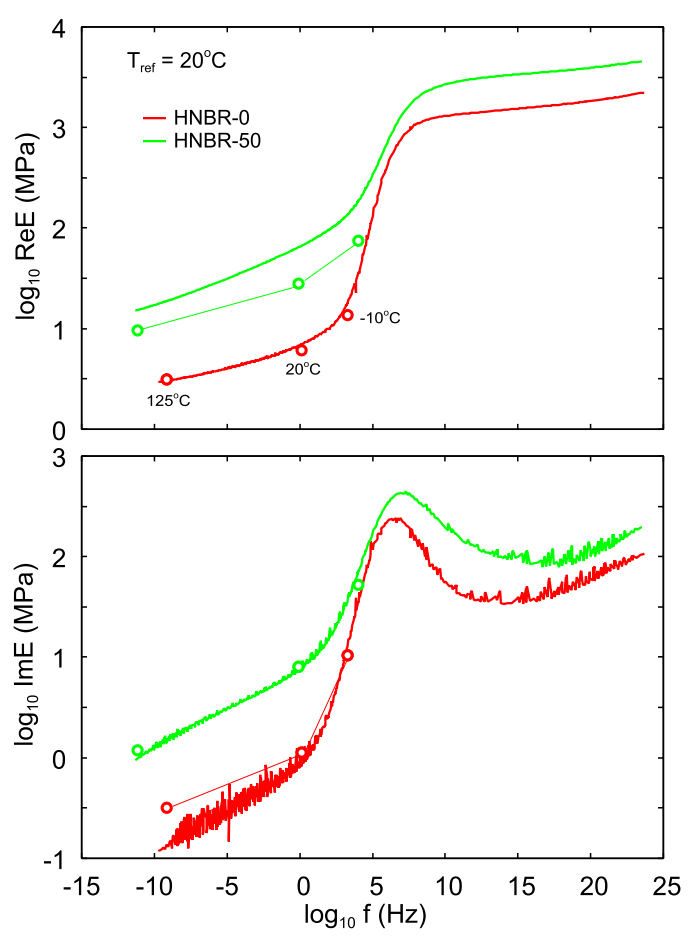

FIG. 24. Thick lines: The viscoelastic modulus master curve for the HNBR0 and HNBR-50 compounds obtained at very low strain (linear response region), $0.04 \%$ strain. Circles: the effective modulus at large strain as obtained for the frequency $f=1 \mathrm{~Hz}$ at different temperatures and then shifted along the frequency axis to the reference temperature $20^{\circ} \mathrm{C}$ using the (low-strain) viscoelastic shift factor $a_{T}$.

in the tension mode, meaning that a strip or a fiber of rubber clamped on both sides is elongated in an oscillatory manner. The complex viscoelastic modulus is first measured in the constant strain mode with a strain amplitude of $0.04 \%$ strain and at different frequencies starting from $28 \mathrm{~Hz}$ and changed in steps until $0.25 \mathrm{~Hz}$ is reached (10 frequency points: $28.0,25.0,14.0$, $7.9,4.4,2.5,1.4,0.79,0.44$, and $0.25 \mathrm{~Hz}$ ). The rather small strain amplitude is chosen in order to avoid strain softening effects, e.g., the Mullins effect or the Payne effect, which can strongly change the viscoelastic response of the rubber specimen. It is not clear how these nonlinear effects would affect the results at different temperatures and one therefore usually measures the low strain master curve in the linear response region. We have found that a strain amplitude of $0.04 \%$ is reasonably good for typical tread rubber compounds. Measuring

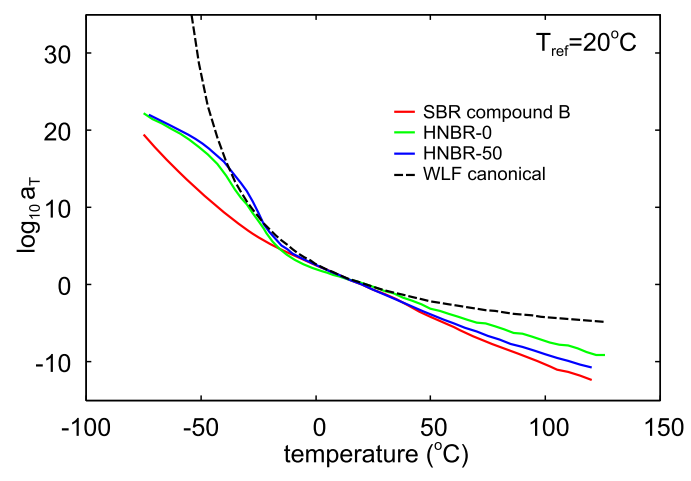

FIG. 25. The shift factor for the SBR compound and the HNBR-0 and HNBR-50 compounds as a function of the temperature. The dashed line is the WLF prediction assuming the canonical coefficients $A=17.44$ and $B=51.6^{\circ} \mathrm{C}$.

the rubber sample in the tension mode also requires prestraining the rubber with a static strain that has to be larger than the dynamic strain during oscillation. The prestrain in the experiments has been set to $0.06 \%$ to avoid compressing the rubber during the DMA measurement.

The experiment usually starts at $-70{ }^{\circ} \mathrm{C}$ and after measuring the modulus at all frequencies mentioned above, the temperature is increased in steps by $5{ }^{\circ} \mathrm{C}$ and the procedure is repeated until $120^{\circ} \mathrm{C}$ is reached. Note that it may be necessary to choose smaller temperature steps when reaching the glass transition temperature $T_{g}$ where the viscoelastic response of the rubber material changes strongly with frequency (and temperature). This makes sure that the curves measured at different temperatures overlap with each other, which is necessary for the shift procedure. The results are then shifted in order to form a smooth $\operatorname{Re} E$ master curve.

To include the non-linear behavior in the characterization of the material, we perform a second experiment which we refer to as the strain sweep experiment to study the softening effects due to, e.g., the breakdown of the filler network (Payne effect). Here the rubber sample is again measured in the oscillation mode at constant temperature but now at a fixed frequency of $1 \mathrm{~Hz}$ while the strain amplitude is increased from very small values $(\approx 0.01 \%)$ until either the sample breaks or the maximum force of the DMA Q800 is reached. We typically reach strain values of order $100 \%$ depending on the temperature (at low temperatures, the maximum strain amplitude may be limited to $\approx 35 \%$ ). The procedure is repeated

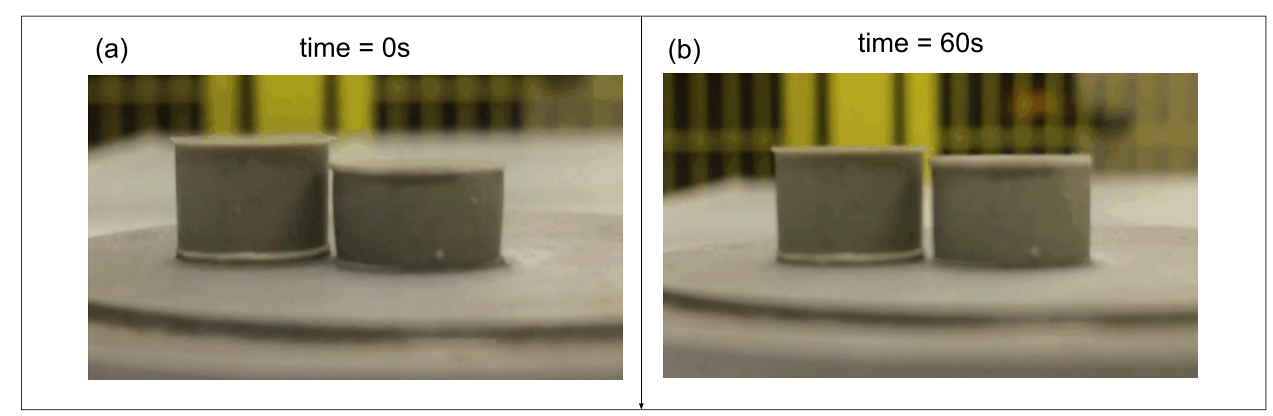

FIG. 26. A rubber cylinder (diameter $1 \mathrm{~cm}$ ) was kept at $T=-30{ }^{\circ} \mathrm{C}$ for 8 days and then compressed by $20 \%$. In (a), we show the uncompressed cylinder (left) and the compressed cylinder (right) immediately after moving the cylinder into room temperature. In (b), we show the uncompressed cylinder (left) and the originally compressed cylinder after $60 \mathrm{~s}$ at room temperature. After even longer time at room temperature, the rubber temperature everywhere approached room temperature and the cylinder recovered its initial shape. 


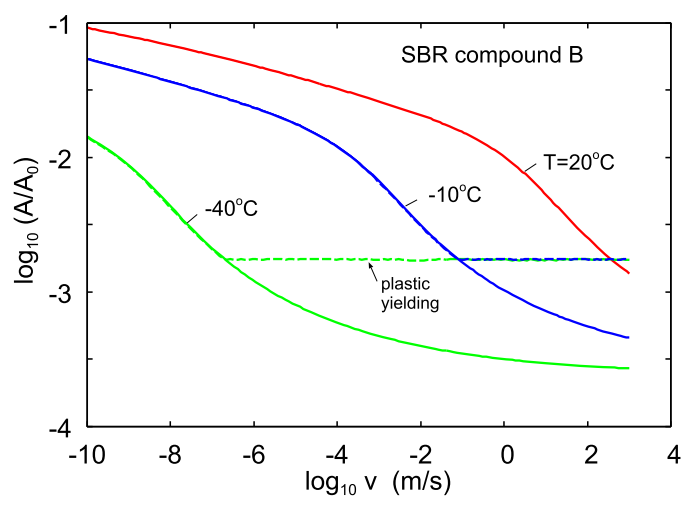

FIG. 27. The normalized contact area $A / A_{0}$ (where $A_{0}$ is the nominal contact area) for the SBR compound as a function of sliding speed (log-log scale) for $T=20^{\circ} \mathrm{C},-10{ }^{\circ} \mathrm{C}$, and $-40{ }^{\circ} \mathrm{C}$. The dashed line is the contact area assuming that the contact pressure in all contact regions equals the yield stress (or penetration hardness) $\sigma_{\mathrm{Y}}=100 \mathrm{MPa}$.

using a new rubber sample for each measurement, at different temperatures.

The thick lines in Fig. 23 show the viscoelastic modulus master curve for the SBR compound obtained at very low strain (linear response region), $0.04 \%$ strain, and for the reference temperature $T_{\text {ref }}=20^{\circ} \mathrm{C}$. In the top panel, we show the real part $\operatorname{Re} E$ and in the bottom panel we show the imaginary part $\operatorname{Im} E$ of the rubber modulus. The circles show the effective modulus at large strain as obtained for the frequency $f=1 \mathrm{~Hz}$ at different temperatures and then shifted along the frequency axis to the reference temperature $20^{\circ} \mathrm{C}$ using the (low-strain) viscoelastic shift factor $a_{T}$. Figure 24 shows similar results for the HNBR-0 and HNBR-50 compounds.

Figure 25 shows the horizontal shift factors for the SBR compound and the HNBR-0 and HNBR-50 compounds as a function of the temperature. The dashed line is the WLF prediction assuming the canonical coefficients $A=17.44$ and $B=51.6^{\circ} \mathrm{C}$. In Table I, we give the glass transition temperature for the rubber compounds. The glass transition temperature is defined as the maximum of $\tan \delta$ as a function of temperature for the frequency $\omega_{0}=0.01 \mathrm{~s}^{-1}$.

\section{Yield stress of HNBR at low temperature}

We have measured the (low temperature) yield stress of filled and unfilled HNBR rubber in both tension (elongation)

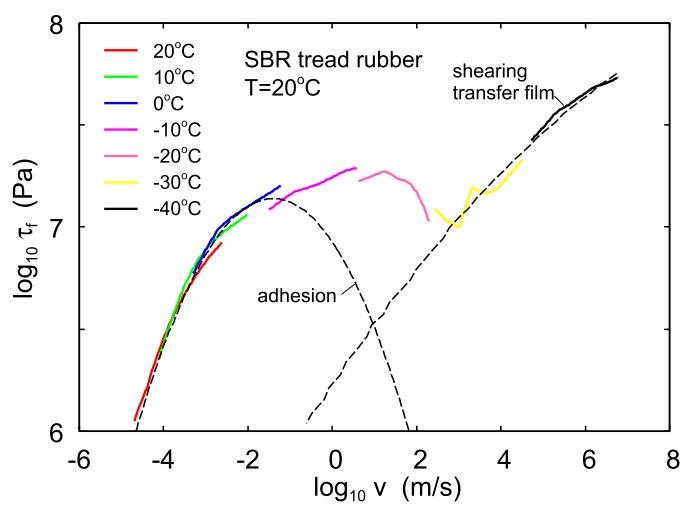

FIG. 28. The frictional shear stress master curve at $T=20{ }^{\circ} \mathrm{C}$ for SBR obtained using the shift factor $a_{T}^{\prime}$ shown in Fig. 11 (red stars). and compression tests. The results in the tension mode are shown in Fig. 20. When the external force was removed, the sample when kept at $T=-40{ }^{\circ} \mathrm{C}$ stayed in its elongated state, but returned to its original undeformed state if the temperature increased above the glass transition temperature. The same was observed in compression tests. Thus, as an example, Fig. 26 shows a rubber cylinder (diameter $1 \mathrm{~cm}$ ) which was first kept at $T=-30{ }^{\circ} \mathrm{C}$ for 8 days, and then compressed by $20 \%$. In (a), we show the uncompressed cylinder (left) and the compressed cylinder (right) immediately after moving the cylinder into room temperature. In (b), we show the uncompressed cylinder (left) and the originally compressed cylinder after $60 \mathrm{~s}$ at room temperature. After even longer time at room temperature, the rubber temperature approaches the room temperature and the cylinder recovers its uncompressed shape.

\section{APPENDIX C: THE FRICTIONAL SHEAR STRESS}

Figure 10 shows the friction master curve as obtained by shifting the $\mu(v)$ velocity segments in Fig. 8 using the shift factor $a_{T}^{\prime}$ shown in Fig. 11 (red stars). For low velocities, where the adhesive contribution gives the dominating velocity dependency, the shifting procedure results in a shift factor as given by (4). However, as discussed in Sec. III, it is not strictly possible to shift the friction coefficient velocity segments directly to obtain a master curve since the shear stress $\tau_{\mathrm{f}}$ has a different temperature dependency than the viscoelastic contribution to the friction and also that of the contact area. Hence one should first calculate the shear stress velocity segments $\tau_{\mathrm{f}}(v)$ from the measured $\mu(v)$ via $\tau_{\mathrm{f}}(v)=p_{0}\left(A_{0} / A\right)\left(\mu(v)-\mu_{\mathrm{visc}}(v)\right)$. The different velocity segments $\tau_{\mathrm{f}}(v)$ obtained for the different temperatures can then be shifted to obtain a smooth master curve for $\tau_{\mathrm{f}}(v)$.

We have obtained the frictional shear stress master curve for all the studied rubber compounds using the procedure described above. When calculating the contact area $A(v)$, we have taken into account that at low temperatures the rubber exhibits a yield stress, so the contact area for low temperatures is given by $A \sigma_{\mathrm{Y}}=A_{0} p_{0}$, where $p_{0}$ is the nominal contact pressure and $\sigma_{\mathrm{Y}}$ is the penetration hardness which we have taken to be $100 \mathrm{MPa}$, i.e., about 3 times higher than the yield stress in elongation as observed for the HNBR rubber. As an example,

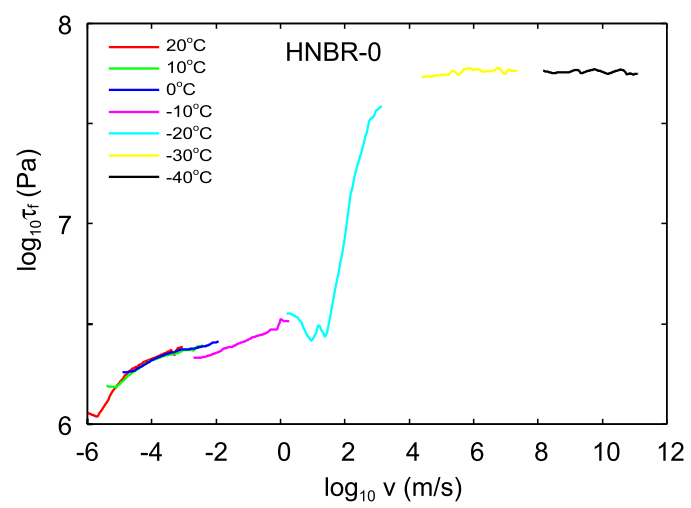

FIG. 29. The frictional shear stress master curve at $T=20^{\circ} \mathrm{C}$ for the HNBR-0 obtained using the shift factor $a_{T}^{\prime}$ shown in Fig. 14 (red stars). 


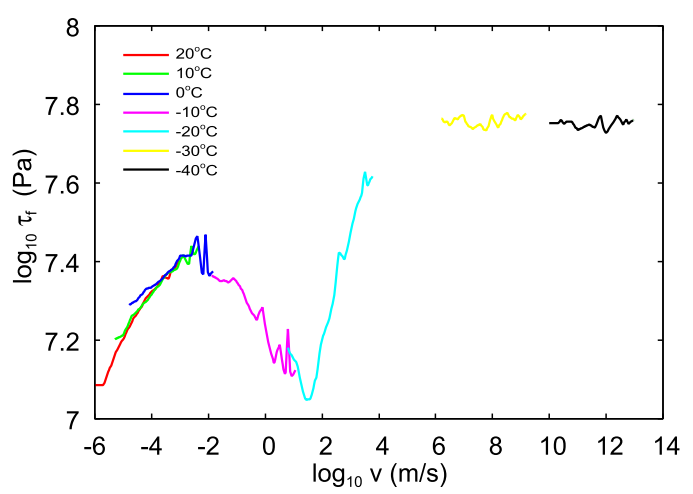

FIG. 30. The frictional shear stress master curve at $T=20^{\circ} \mathrm{C}$ for the HNBR50 obtained using the shift factor $a_{T}^{\prime}$ shown in Fig. 16 (red stars).

in Fig. 27, we show for the SBR compound the normalized contact area $A / A_{0}$ (where $A_{0}$ is the nominal contact area) as a function of sliding speed (log-log scale) for $T=20{ }^{\circ} \mathrm{C}$, $-10^{\circ} \mathrm{C}$, and $-40^{\circ} \mathrm{C}$. The dashed line is the contact area assuming that the contact pressure in all contact regions equals the penetration hardness $\sigma_{\mathrm{Y}}=100 \mathrm{MPa}$.

In Fig. 28, we show the frictional shear stress master curve at $T=20{ }^{\circ} \mathrm{C}$ for the SBR compound. At low sliding speeds (corresponding to high temperatures), the velocity segments have been shifted with the Arrhenius shift factor (4) while at high sliding speeds the shift factor is similar to the bulk shift factor $a_{T}$. This is shown in Fig. 11 where the red stars are the shift factor $a_{T}^{\prime}$ used to obtain the master curve in Fig. 28 while the green squares are the bulk viscoelastic shift factor $a_{T}$. The dashed line is the Arrhenius shift factor (4).

The dashed lines in Fig. 28 indicate the adhesive and transfer film contributions to the frictional shear stress. The adhesive contribution is given by (3) and depends on temperature according to the Arrhenius law (4) while the contribution from shearing the transfer film has the same temperature dependency as the bulk viscoelastic modulus.

In Figs. 29 and 30, we show the frictional shear stress master curves for the unfilled and filled HNBR compounds, and Figs. 14 and 16 shows the corresponding shift factors. At the three highest temperatures, the shift factor obeys the Arrhenius law (4). For the two lower temperatures, the shear stress is nearly velocity independent and it is not possible to determine the temperature dependency of the shift

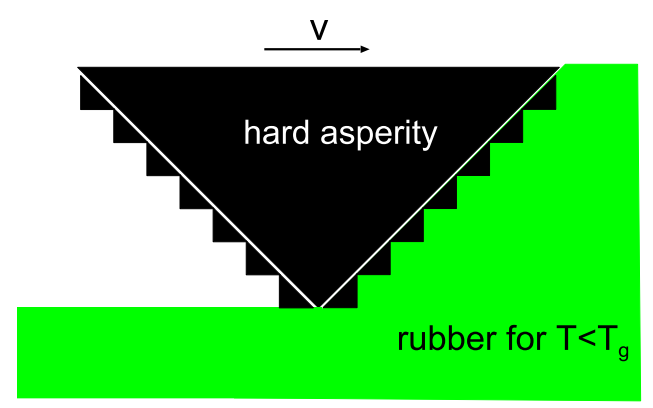

FIG. 31. A hard asperity plastically plowing rubber (at a temperature well below the rubber glass transition temperature). The "big" asperity has smaller asperities on top of it but they have a negligible influence on the plowing force since they have only a small influence on the asperity plowing cross section (schematic). factor from the measured data and in the figures we have just assumed that it is the same as the bulk viscoelastic shift factor.

\section{APPENDIX D: RUBBER FRICTION AND WEAR AT LOW TEMPERATURES}

Consider the contact between a rigid solid with random roughness and an elastic solid with a flat surface. If we consider the rough surface at the magnification $\zeta$, we observe only the roughness components with wavenumber $q<q_{0} \zeta$, where $q_{0}$ is some reference wave number, e.g., the roll-off wavenumber of the surface roughness power spectrum. The surface rms slope $h^{\prime}$ will increase as we increase the magnification $\zeta$. As long as the contact is elastic, the relative contact $\operatorname{area}^{9,42-44}$

$$
\frac{A}{A_{0}} \approx \frac{2 p_{0}}{h^{\prime} E},
$$

where $A_{0}$ is the nominal contact area and $p_{0}$ is the applied nominal contact pressure. The average pressure in the asperity contact regions $p=p_{0} A_{0} / A \approx h^{\prime} E / 2$. We assume that when $p$ reaches the yield stress or penetration hardness $p_{\mathrm{Y}}$, the contact deforms plastically. This will occur in the contact regions observed at the magnification where $p_{\mathrm{Y}} \approx h^{\prime} E / 2$, where $p_{\mathrm{Y}}$ is the rubber penetration hardness. Thus, a plastic flow will occur in the asperity contact regions at the magnification $\zeta^{*}$, where including all the roughness with wavenumber $q<q_{0} \zeta^{*}$ results in a rms slope $h^{\prime} \approx 2 p_{\mathrm{Y}} / E$. Assuming the penetration hardness to be $\sim 3$ times higher than the yield stress in elongation, we get from Fig. 20 that $p_{\mathrm{Y}} \approx 100 \mathrm{MPa}$ and using (in the glassy region) $E \approx 2 \times 10^{3} \mathrm{MPa}$ we get $h^{\prime} \approx 0.1$. For the concrete surface, we use below this rms slope corresponding to the wavenumber $q=\zeta^{*} q_{0} \approx 1 \times 10^{3} \mathrm{~m}^{-1}$.

To summarize, the roughness with wavenumber $q<\zeta^{*} q_{0}$ will deform the rubber elastically and will not contribute to the friction. The roughness components with wavenumber $q>\zeta^{*} q_{0}$ will plastically deform the rubber and will give a plowing contribution to the friction as a result of the unbalanced tangential force acting on the asperity (see Fig. 31). However, for surfaces with fractal dimension $D_{\mathrm{f}}$ close to 2 (as is typical for most real surfaces), the short wavelength roughness is not very important for the plowing contribution to the friction. This can be seen as follows:

Let us "discretize" the roughness and assume that instead of a continuous distribution of roughness components, there is roughness only with discrete wavenumbers, say $q_{0}, 10 q_{0}$, $100 q_{0}, \ldots$. In this case, on top of an asperity with linear size $\lambda$, there will be smaller asperities with linear size $\lambda / 10$ (and so on), as indicated in Fig. 31. Now consider the asperity with the linear size $\lambda^{*}$ corresponding to the wavenumber $q=q_{0} \zeta^{*}$. On top of this occur asperities with smaller size $\lambda=\lambda^{*} / 10$. If the fractal dimension $D_{\mathrm{f}}=2$, these asperities are self-similar to the original asperity, i.e., the ratio between the height and width of it is the same as for the asperity observed at the magnification $\zeta^{*}$. Thus the plowing cross section area is only slightly modified by the smaller asperities observed when the magnification is increased beyond $\zeta^{*}$. That is, in a first approximation, we can assume that the plowing friction is determined only by the (smallest) asperities observed at the magnification 
$\zeta^{*}$. At this magnification, the rms slope of the roughness is $h^{\prime} \approx 2 p_{\mathrm{Y}} / E$.

The argument presented above is not valid if the fractal dimension is much higher than 2. Thus if $D_{\mathrm{f}}=3$ (as for frozen capillary waves), the "new" roughness observed as the magnification increases has the same amplitude as the roughness observed at a lower magnification, and in this case one would need to include all the roughness components when estimating the plowing contribution to the friction force.

When asperities in a narrow region of length scales give the main contribution to the plowing friction (as is the case when $D_{\mathrm{f}} \approx 2$ ), we can use the model of Tabor and Bowden, which only considers asperities on a single length scale, to estimate the plowing friction. Thus, the plastic plowing gives a contribution to the friction given by $\mu \approx \alpha h^{\prime}$, where $\alpha$ is a number $<1$ but of order unity which depends on the shape of the asperities. For example, for a conical asperity, $\alpha=2 / \pi$. Using this value for $\alpha$ and $h^{\prime}=0.1$ gives a contribution to the friction coefficient $\Delta \mu \approx 0.06$, which is much smaller than the observed value $\approx 0.6$. This estimation is very rough, and there is a need for developing a more accurate approach to the plowing contribution for surfaces with roughness on many length scales, but the deviation from the measured value is so large that we conclude that there must be other processes which give the main part of the friction at low temperatures.

As stated above, most of the rubber plastic deformations are reversible and disappear as the rubber heats up above the glass transition temperature. Thus, we do not expect any strong wear tracks to remain on the rubber surface at room

(a) after sliding at $\mathrm{T}=20^{\circ} \mathrm{C}$

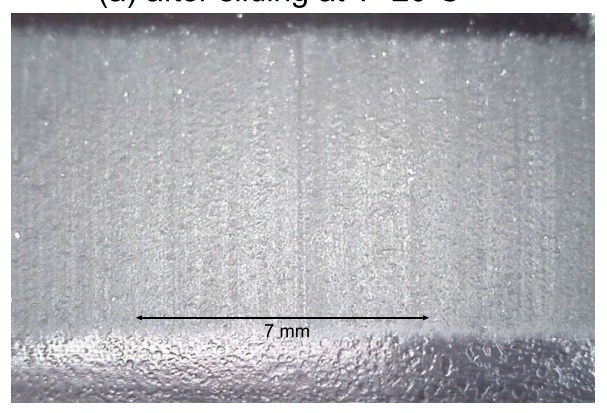

(b) after sliding at $\mathrm{T}=-40^{\circ} \mathrm{C}$ and cleaned

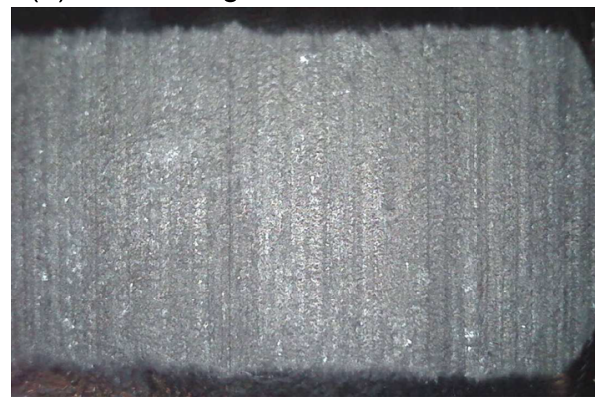

FIG. 32. (a) The surface of the HNBR-50 cylinder after sliding $320 \mathrm{~cm}$ on the concrete surface at $T=20{ }^{\circ} \mathrm{C}$ at the nominal contact pressure $p_{0}=0.40 \mathrm{MPa}$. (b) The same surface after sliding $80 \mathrm{~cm}$ on the concrete surface at $T=-40{ }^{\circ} \mathrm{C}$ at the nominal contact pressure $p_{0}=0.92 \mathrm{MPa}$. The latter surface was "cleaned" by removing rubber and concrete wear particles using adhesive tape. In both cases, the sliding speed $v=1 \mathrm{~mm} / \mathrm{s}$.

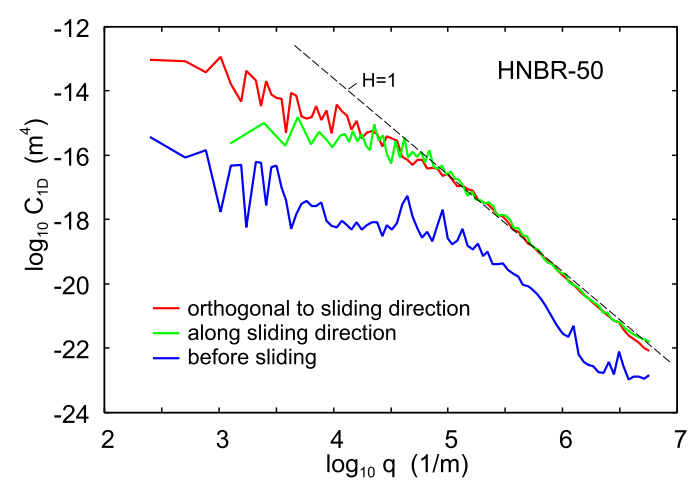

FIG. 33. The one-dimensional (1D) surface roughness power spectrum of the HNBR-50 cylinder surface before (blue line) and after (red and green lines) sliding at $T=-40^{\circ} \mathrm{C}$ on the concrete surface [see Fig. 32(b)]. The red line is the $1 \mathrm{D}$ power spectrum orthogonal to the sliding direction and the green line along the sliding direction.

temperature, unless the substrate has very sharp asperities (like sandpaper) which can break covalent bonds in the rubber. This is consistent with optical pictures of the contact (see Fig. 32) where only very weak (shallow) wear tracks can be observed.

We have performed stylus topography measurements along and orthogonal to the sliding direction of the rubber surface shown in Fig. 32(b). Figure 33 shows the one-dimensional (1D) surface roughness power spectrum before (blue line) and after (red and green lines) sliding at $T=-40^{\circ} \mathrm{C}$ [see Fig. 32(b)]. The red line is the $1 \mathrm{D}$ power spectrum orthogonal to the sliding direction and the green line along the sliding direction. We note that there is a slight increase in the surface roughness power spectrum at wavenumber $q<10^{4} \mathrm{~m}^{-1}$ for the direction orthogonal to the sliding direction as compared to parallel to the sliding direction. However, the short wavelength roughness, down to length scales of order a few micrometer (which is the lateral resolution of our stylus instrument), appears to be almost identical in the two directions. This remarkable result indicates that the wear process at short length scales, which is the origin of the small rubber wear particles produced during sliding (see Fig. 35), results in a surface with nearly isotropic roughness at short length scale.

Figure 34 shows the height probability distribution of the HNBR-50 cylinder surface after sliding at $T=-40{ }^{\circ} \mathrm{C}$ [see

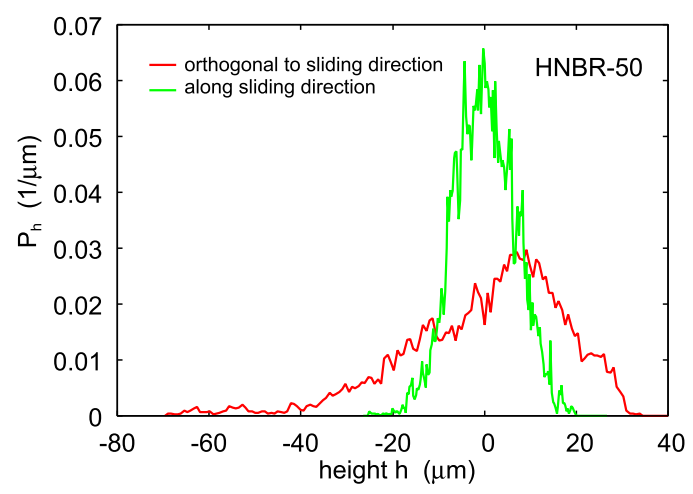

FIG. 34. The height probability distribution of the HNBR-50 cylinder surface after sliding at $T=-40{ }^{\circ} \mathrm{C}$ on the concrete surface [see Fig. 32(b)]. The red line is the $1 \mathrm{D}$ power spectrum orthogonal to the sliding direction and the green line along the sliding direction. 


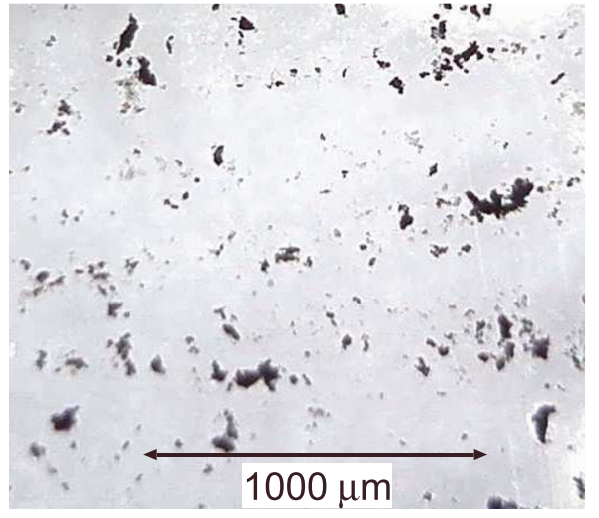

FIG. 35. Rubber wear particles adhering to adhesive tape, produced during sliding at $v=1 \mathrm{~mm} / \mathrm{s}$ (for $80 \mathrm{~cm}$ ) of the HNBR-50 compound on the concrete surface at $T=-40^{\circ} \mathrm{C}$. The nominal contact pressure $p_{0}=0.92 \mathrm{MPa}$.

Fig. 32(b)]. The red line is the height distribution orthogonal to the sliding direction and the green line along the sliding direction. As expected from the power spectra, the amplitude of the corrugation in the profile is the largest in the direction orthogonal to the sliding direction.

Figure 35 shows rubber wear particles obtained by squeezing an adhesive tape against the surface in Fig. 32(b). Most of the rubber particles are very small, less than $\sim 30 \mu \mathrm{m}$, and most likely the big rubber particles are an agglomeration of many small (say micrometer) rubber wear particles. Thus the rubber wear appears to involve much smaller length scales than the length scale (of order 01-1 mm) where plastic deformation of the rubber occurs. This again is supported by the observation that most of the plastic deformations disappear when the temperature is increased above the rubber glass transition temperature.

Since the rubber elastic modulus becomes very high at low temperatures, the stresses acting on the substrate surface (here concrete) at temperatures below the rubber glass transition temperature are very high. As a result, we observe wear fragments broken off from the concrete and transferred to the rubber surface. Thus, Fig. 36 shows fragments (white regions) from the concrete surface which are adhering to the

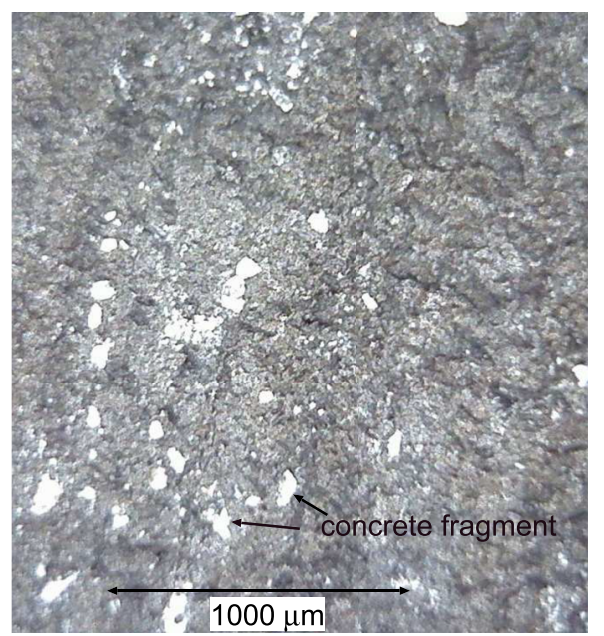

FIG. 36. The rubber surface shown in Fig. 32(b) before removing rubber and concrete wear particles. The white particles are fragments from the concrete surface. rubber surface after the rubber was slid on the concrete surface at $T=-40{ }^{\circ} \mathrm{C}$.

We have shown above that most of the rubber wear particles are very small and not associated with the plastic deformation of the rubber which occurs at much longer length scales. Consider now the energy dissipated during sliding the distance $L$. We can write this as $\mu F_{\mathrm{N}} L=\mu A p_{\mathrm{Y}} L$. We assume that the sliding results in the removal of small rubber particles. Removing this material requires breaking covalent bonds. Let $\gamma$ be the energy per unit surface area to break the bonds. This is also the energy to propagate an opening crack in the rubber and depends on the crack tip speed. ${ }^{45,46}$ Let $R$ be the average radius of the asperity contact regions observed at the magnification where the contact pressure equals the plastic yield stress (see above). During sliding the distance $L$, the surface area covered by the contact regions will be $N_{0} 2 R L$, where $N_{0}$ is the number of contact regions so that $N_{0} \pi R^{2}=A$. We consider now the limiting case where rubber wear particles are removed everywhere within the area $N_{0} 2 R L$. In this case, the number of formed rubber particles equals $N=N_{0} 2 R L /\left(\pi r^{2}\right)$. This gives the volume of rubber removed $v \approx N\left(4 \pi r^{3} / 3\right)$ which requires the energy $U \approx N 4 \pi r^{2} \gamma$. The contribution to the friction force from this wear process would be

$$
\mu=\frac{U}{A p_{\mathrm{Y}} L}=\frac{8 \gamma}{\pi R p_{\mathrm{Y}}} .
$$

Using typical numbers $R=0.1 \mathrm{~mm}, \gamma=30 \mathrm{~J} / \mathrm{m}^{2}$, and $p_{\mathrm{Y}}=100 \mathrm{MPa}$, we get $\mu \approx 0.01$ which is negligible. Thus neither plastic plowing nor the wear process can explain the observed friction coefficient in the low temperature sliding region.

${ }^{1}$ J. A. Greenwood and D. Tabor, "The friction of hard sliders on lubricated rubber: The importance of deformation losses," Proc. Phys. Soc. 71, 989 (1958).

${ }^{2}$ A. Schallamach, "How does rubber slide," Wear 17, 301 (1971).

${ }^{3}$ A. D. Roberts and D. Tabor, Proc. R. Soc. A 325, 323 (1971); A. D. Roberts, in The Physics of Tire Friction: Theory and Experiment, edited by D. F. Hays and A. L. Browne (Plenum, New York, 1974).

${ }^{4}$ K. A. Grosch, Proc. R. Soc. London, Ser. A 274, 21 (1963).

${ }^{5}$ K. A. Grosch, Rubber Chem. Technol. 69, 495 (1997).

${ }^{6}$ M. Klüppel and G. Heinrich, Rubber Chem. Technol. 73, 578 (2000).

${ }^{7}$ L. Busse, I. Boubakri, and M. Klüppel, "Friction master curves for rubber on dry and wet granite: Experiments and simulations," KGK Kautschuk, Gummi, Kunststoffe 64(5), 35 (2011).

${ }^{8}$ B. N. J. Persson, Surf. Sci. 401, 445 (1998).

${ }^{9}$ B. N. J. Persson, J. Chem. Phys. 115, 3840 (2001).

${ }^{10}$ M. Scaraggi and B. N. J. Persson, "Friction and universal contact area law for randomly rough viscoelastic contacts," J. Phys.: Condens. Matter 27, 105102 (2015).

${ }^{11}$ R. Bugnicourt, P. Sainsot, N. Lesaffre, and A. A. Lubrecht, "Transient frictionless contact of a rough rigid surface on a viscoelastic half-space," Tribol. Int. 113, 279 (2017).

${ }^{12}$ A. Tiwari, L. Dorogin, M. Tahir, K. W. Stockelhuber, G. Heinrich, N. Espallargas, and B. N. J Persson, "Rubber contact mechanics: Adhesion, friction and leakage of seals," Soft Matter 13, 9103 (2017).

${ }^{13}$ A. L. Gal and M. Klüppel, "Investigation and modelling of rubber stationary friction on rough surfaces," J. Phys.: Condens. Matter 20, 015007 (2007).

${ }^{14}$ A. Lang and M. Klüppel, "Influences of temperature and load on the dry friction behaviour of tire tread compounds in contact with rough granite," Wear 380-381, 15 (2017).

${ }^{15}$ B. Lorenz, B. N. J. Persson, G. Fortunato, M. Giustiniano, and F. Baldoni, J. Phys.: Condens. Matter 25, 095007 (2013).

${ }^{16}$ O. Bäumchen, J. D. McGraw, J. A. Forrest, and K. Dalnoki-Veress, "Reduced glass transition temperatures in thin polymer films: Surface effect or artifact?,” Phys. Rev. Lett. 109, 055701 (2012). 
${ }^{17}$ S. Peter, H. Meyer, and J. Baschnagel, "Thickness-dependent reduction of the glass-transition temperature in thin polymer films with a free surface," J. Polym. Sci., Part B: Polym. Phys. 44, 2951 (2006).

${ }^{18}$ K. G. Rowe, A. I. Bennett, B. A. Krick, and W. G. Sawyer, "In situ thermal measurements of sliding contacts," Tribol. Int. 62, 208 (2013).

${ }^{19}$ B. N. J. Persson, Tribol Lett. 54, 99 (2014).

${ }^{20}$ B. N. J. Persson, "Author response to the comment by popov on "contact mechanics for randomly rough Surfaces: On the validity of the method of reduction of dimensionality," Tribol. Lett. 60, 33 (2015); A. Lyashenko, L. Pastewka, and B. N. J. Persson, "Comment on "friction between a viscoelastic body and a rigid surface with random self-affine roughness," Phys. Rev. Lett. 111, 189401 (2013).

${ }^{21}$ B. N. J. Persson, B. Lorenz, M. Shimizu, and M. Koishi, "Multiscale contact mechanics with application to seals and rubber friction on dry and lubricated surfaces," in Designing of Elastomer Nanocomposites: From Theory to Applications, Advances in Polymer Science (Springer, 2016), p. 103.

${ }^{22}$ A. K. Bhowmick, C. Neogi, and P. Nasu, "Threshold tear strength of carbon black filled rubber vulcanizates," Appl. Polym. 41, 917 (1990).

${ }^{23}$ B. Lorenz, Y. R. Oh, S. K. Nam, S. H. Jeon, and B. N. J. Persson, "Rubber friction on road surfaces: Experiment and theory for low sliding speeds," J. Chem. Phys. 142, 194701 (2015).

${ }^{24}$ A. Schallamach, Wear 6, 375 (1963).

${ }^{25}$ Y. B. Cherniak and A. I. Leonov, Wear 108, 105 (1986); A. I. Leonov, ibid. 141, 137 (1990).

${ }^{26}$ B. N. J. Persson and A. I. Volokitin, Eur. Phys. J. E 21, 69 (2006).

${ }^{27}$ Chateauminois and Fretigny (see Ref. 28) found the frictional shear stress on smooth and slightly (atomically) roughened glass to be $\approx 0.1 \mathrm{MPa}$ and $\approx 0.2 \mathrm{MPa}$, respectively. These authors also found that the shear stress is independent of the nominal contact pressure, as expected for soft dry contacts, where the contact pressure is small, typically of order $1 \mathrm{MPa}$ or less. The magnitude of $\tau_{\mathrm{f}}$ for PDMS is much smaller than for most other types of rubber. This reflects the inert (low surface energy) nature of PDMS.

${ }^{28}$ A. Chateauminois and C. Fretigny, "Local friction at a sliding interface between an elastomer and a rigid spherical probe," Eur. Phys. J. E 27, 221 (2008).

${ }^{29}$ B. Schmidtke, M. Hofmann, A. Lichtinger, and E. A. Rössler, "Temperature dependence of the segmental relaxation time of polymers revisited," Macromolecules 48, 3005 (2015).

${ }^{30}$ S. Herminghaus, K. Jacobs, and R. Seemann, "The glass transition of thin polymer films: Some questions, and a possible answer," Eur. Phys. J. E 5, 531 (2001).
${ }^{31}$ B. Zuo, Y. Liu, Y. Liang, D. Kawaguchi, K. Tanaka, and X. Wang, "Glass transition behavior in thin polymer films covered with a surface crystalline layer," Macromolecules 50, 2061 (2017).

${ }^{32}$ J. A. Forrest and K. Dalnoki-Veress, "The glass transition in thin polymer films," Adv. Colloid Interface Sci. 94, 167 (2001).

${ }^{33}$ P. G. de Gennes, "Glass transitions in thin polymer films," Eur. Phys. J. E 2, 201 (2000).

${ }^{34}$ K. Fukao and Y. Miyamoto, "Glass transition temperature and dynamics of $\alpha$-process in thin polymer films," Europhys. Lett. 46, 649 (1999).

${ }^{35}$ P. G. Whitten and H. R. Brown, Phys. Rev. E 76, 026101 (2007).

${ }^{36}$ I. M. Sivebaek, V. N. Samoilov, and B. N. J. Persson, "Frictional properties of confined polymers," Eur. Phys. J. E 27, 37 (2008).

${ }^{37}$ B. N. J. Persson, J. Phys.: Condens. Matter 18, 7789 (2006).

${ }^{38}$ G. Fortunato, V. Ciaravola, A. Furno, B. Lorenz, and B. N. J. Persson, J. Phys. C: Condens. Matter 27, 175008 (2015).

${ }^{39}$ B. N. J. Persson, Tribol. Lett. 56, 77 (2014).

${ }^{40}$ B. N. J. Persson, O. Albohr, U. Tartaglino, A. I. Volokitin, and E. Tosatti, J. Phys.: Condens. Matter 17, R1 (2005).

${ }^{41}$ G. Carbone, B. Lorenz, B. N. J. Persson, and A. Wohlers, Eur. Phys. J. E 29, 275 (2009).

${ }^{42}$ M. H. Müser, W. B. Dapp, R. Bugnicourt, P. Sainsot, N. Lesaffre, T. A. Lubrecht, B. N. J. Persson, K. Harris, A. Bennett, K. Schulze, S. Rohde, P. Ifju, W. G. Sawyer, T. Angelini, H. A. Esfahani, M. Kadkhodaei, S. Akbarzadeh, J.-J. Wu, G. Vorlaufer, A. Vernes, S. Solhjoo, A. I. Vakis, R. L. Jackson, Y. Xu, J. Streator, A. Rostami, D. Dini, S. Medina, G. Carbone, F. Bottiglione, L. Afferrante, J. Monti, L. Pastewka, M. O. Robbins, and J. A. Greenwood, "Meeting the contact-mechanics challenge," Tribol. Lett. 65, 118 (2017).

${ }^{43}$ S. Hyun and M. O. Robbins, "Elastic contact between rough surfaces: Effect of roughness at large and small wavelengths," Tribol. Int. 40, 1413 (2007).

${ }^{44}$ N. Prodanov, W. B. Dapp, and M. H. Müser, "On the contact area and mean gap of rough, elastic contacts: Dimensional analysis, numerical corrections, and reference data," Tribol. Lett. 53, 433 (2013).

${ }^{45}$ B. N. J. Persson and E. A. Brener, Phys. Rev. E 71, 036123 (2005).

${ }^{46}$ B. N. J. Persson, O. Albohr, G. Heinrich, and H. Ueba, J. Phys.: Condens. Matter 17, R1071 (2005).

${ }^{47}$ A. Almqvist, C. Campana, N. Prodanov, and B. N. J. Persson, J. Mech. Phys. Solids 59, 2355 (2011).

${ }^{48} \mathrm{G}$. Reiter, private communication (2018). 